\title{
Evaluaciones económicas de intervenciones dirigidas a la prevención, tratamiento y/o rehabilitación de trastornos por consumo de alcohol: una revisión sistemática
}

\author{
Economic evaluations of interventions aimed at \\ the prevention, treatment and/or rehabilitation of \\ alcohol-related disorders: a systematic review
}

\author{
Marta Trapero-Bertran*, Dolors Gil-Doménech**, Ana Magdalena Vargas-Martínez***. \\ * Departamento de Ciencias Básicas. Instituto Universitario de Pacientes. Universitat Internacional de Catalunya (UIC Barcelona). \\ ** Facultad de Ciencias Económicas y Sociales. Universitat Internacional de Catalunya (UIC Barcelona). \\ *** Departamento de Enfermería. Facultad de Enfermería, Fisioterapia y Podología. Universidad de Sevilla (US). Universitat \\ Internacional de Catalunya (UIC Barcelona).
}

\begin{abstract}
Resumen
El objetivo de esta revisión sistemática de la literatura es identificar evaluaciones económicas de programas o intervenciones dirigidas a la prevención, tratamiento y rehabilitación de trastornos por consumo de alcohol, así como determinar aquellos tipos de programas, tratamientos o intervenciones que son eficientes. Se realizó una revisión sistemática de la literatura mediante la búsqueda en las siguientes bases de datos: National Health Service Economic Evaluation Database (NHS EED), Health Technology Assessment (HTA), MEDLINE Ovid and PubMed. Los términos de búsqueda utilizados fueron en inglés. No se aplicó ninguna restricción de tiempo. Se utilizó un formulario de extracción de datos para resumir la información. La revisión sistemática siguió las recomendaciones (PRISMA-P) sobre la presentación de informes de revisiones sistemáticas. Las intervenciones fueron clasificadas en tres categorías: «A» tratamientos para personas con trastornos por consumo de alcohol (prevención terciaria); «B» tratamientos para personas en riesgo de problemas relacionados con el alcohol (prevención secundaria); «C» legislación sobre políticas e intervenciones de aplicación (prevención terciaria). Además, las intervenciones «A» fueron subclasificadas en intervenciones psicológicas, farmacológicas y combinadas. Se incluyeron 63 documentos. En términos de tratamientos para personas con trastornos por uso de alcohol, cualquier intervención psicosocial en comparación con ninguna intervención parece ser una estrategia dominante. En términos de tratamientos para personas en riesgo de problemas relacionados con el alcohol, la intervención breve parece ser dominante o rentable en comparación con no hacer nada. Los controles publicitarios, las subidas de impuestos, las licencias, la edad legal para consumir alcohol y las campañas en los medios de comunicación parecen ser una estrategia dominante o rentable en comparación con ninguna intervención o prueba aleatoria de alcoholemia. Se han ampliado las revisiones anteriores al mostrar los programas de alcohol según criterios de eficiencia. A pesar de ello, los estudios disponibles al respecto tienen enfoques heterogéneos y la mayoría no define adecuadamente los costes incluidos en su análisis. Por tanto, es necesario continuar evaluando en términos de eficiencia este tipo de intervenciones para informar mejor la toma de decisiones en salud pública.

Palabras clave: alcohol, revisión sistemática, eficiencia, coste-efectividad,
\end{abstract} clasificación intervenciones.

\begin{abstract}
The aim of this systematic literature review is to identify economic evaluations of programmes or interventions aimed at the prevention, treatment and rehabilitation of alcohol use disorders, as well as to determine those types of programmes, treatments or interventions that are efficient. The systematic literature review was conducted by searching the following databases: National Health Service Economic Evaluation Database (NHS EED), Health Technology Assessment (HTA), MEDLINE Ovid and PubMed. The search terms used were in English. No time restriction was applied. A data extraction form was used to draw information. The systematic review follows the recommendations of the Preferred Reporting Items for Systematic Review and Meta-Analysis Protocols (PRISMA-P) on reporting systematic reviews. The interventions were classified into three categories: "A" treatments for people with alcohol use disorders (tertiary prevention); "B" treatments for people at risk for alcohol-related problems (secondary prevention); "C" policy legislation and enforcement interventions (primary prevention). Furthermore, the "A" interventions were subclassified into psychological, pharmacological and combined interventions. The review included 63 papers. In terms of treatments for people with alcohol use disorders, any psychosocial intervention compared to no intervention appeared to be a dominant strategy. In terms of treatments for people at risk of alcohol-related problems, brief intervention appears to be dominant or cost-effective when compared to no intervention. Advertising controls, tax increases, licensing, legal drinking age, and mass media campaigns seem to be dominant or cost-effective strategies compared to no intervention or random breath testing. Previous reviews have been extended by depicting alcohol programmes according to their efficiency. Despite this, the available studies in this regard have heterogeneous approaches and most do not adequately define the costs included in their analyses. Therefore, it is necessary to encourage the evaluation of the efficiency of these types of interventions to aid decision-making in public health. Key words: alcohol, systematic review, efficiency, cost-effectiveness, intervention classification.
\end{abstract}


E 1 consumo de alcohol es uno de los principales factores de riesgo de mortalidad y discapacidad en todo el mundo, lo que representa el 5,3\% de todas las muertes y el 5,1 \% de todos los años de vida ajustados en función de la discapacidad (AVAD) a nivel mundial (Organización Mundial de la Salud, 2018). Smyth et al. (2015) confirmaron que el abuso de alcohol se asoció con un mayor riesgo de mortalidad, cáncer y lesiones y un riesgo reducido no significativo de infarto agudo de miocardio. La cantidad y el patrón de consumo de alcohol pueden tener diferentes asociaciones con los resultados y los costes para la salud. Hace tres décadas, Burke (1988) ya había estimado el impacto económico del trastorno por uso de alcohol (TUA) y el alcoholismo, cuantificando pérdidas de miles de millones de dólares anuales debido a la pérdida de productividad y empleo. Rehm et al. (2009) afirmaron que los costes asociados con el alcohol ascienden a más del $1 \%$ del producto interior bruto en los países de ingresos altos y medios, y que los costes del daño social constituyen una proporción importante además de los costes de salud y que deben aumentarse de manera urgente las acciones para reducir la carga y los costes asociados con el alcohol.

La Evaluación Económica (EE) es la herramienta más relevante para los tomadores de decisiones en salud (Goeree y Diaby, 2013) para comparar posibles alternativas de acción tanto en términos de costes como de resultados para la salud. Hay cuatro tipos diferentes de EE cuya diferencia principal radica en su forma de medir, valorar e incluir los resultados en el análisis (Drummond, Sculpher, Claxton, Stoddard y Torrance, 2015). Con el Análisis de coste-efectividad (ACE), las alternativas se comparan en términos de costes y resultados, y los resultados se miden y valoran en unidades naturales recopiladas en ensayos clínicos o estudios observacionales. En un Análisis de coste-utilidad (ACU), se calculan los costes y resultados esperados para cada intervención, con la medida de resultado expresada principalmente como años de vida ajustados por calidad (AVAC), que combina la calidad de la duración y la duración de la vida en una sola medida (Drummond et al., 2015). Los resultados de una EE se presentan en términos de una ratio de coste-efectividad incremental (RCEI), que es literalmente las diferencias en los costes medios esperados divididos por la diferencia en los resultados medios esperados (Drummond et al., 2015). El RCEI proporciona una medida del coste esperado necesario para obtener una unidad de efecto. Cuando un programa o una intervención mejora los resultados y reduce (ahorra) costes, se considera entonces que existe dominancia (Drummond et al., 2015). Durante los últimos años, las evaluaciones económicas han ganado importancia como fuente de información para los tomadores de decisiones en el campo de la salud pública (Drummond et al., 2007; Williams, McIver, Moore y Bryan, 2008). Se ha mostrado que el mercado por sí mismo no logra soluciones eficientes, por lo que los tomadores de decisiones juegan un papel clave porque pueden decidir cómo asignar los recursos escasos (Drummond et al., 2015; Gold, Siegel, Russell y Weinstein, 1996; Kernick, 2003; Sloan y Hsieh, 2012). Por tanto, para reducir la carga de enfermedades y lesiones asociadas con el consumo de alcohol, es importante identificar las intervenciones rentables para apoyar las estrategias e iniciativas nacionales de salud para reducir el consumo nocivo de alcohol.

Donaldson, Mugford y Vale (2002) defendieron el valor de las revisiones sistemáticas de las evaluaciones económicas como herramienta para promover la atención sanitaria basada en la evidencia. Sugieren que el valor de la revisión sistemática de la evidencia de la evaluación económica no es generar un único resultado o recomendación de autoridad en términos de rentabilidad relativa, sino más bien ayudar a los tomadores de decisiones a entender la estructura del problema de la asignación de recursos y los impactos potenciales.

Se han publicado algunas revisiones sistemáticas, evaluando diferentes tipos de programas, estrategias o intervenciones, tales como terapias psicológicas (Meads, Ting, Dretzke y Bayliss, 2007) y tratamientos farmacológicos (Ndegwa y Cunningham, 2009) desde un punto de vista de la eficiencia, tratando de identificar cuál de los servicios nacionales de salud (NHS, National Health Service) debería promoverse para reducir el consumo de alcohol. Además, se han publicado otras revisiones sistemáticas de la eficiencia, tales como de las campañas en los medios de comunicación para reducir la conducción bajo los efectos del alcohol y los accidentes de tráfico relacionados con el alcohol (Yadav y Kobayashi, 2015), las intervenciones de farmacéuticos comunitarios o las intervenciones de tratamiento del uso de alcohol (Brown et al., 2016), destacando las más eficientes en términos de resultados de salud. Además, Barbosa, Godfrey y Parrott (2010) realizaron una revisión de la metodología adoptada en evaluaciones económicas previas del tratamiento de problemas relacionados con el alcohol, y ofrecieron recomendaciones de investigación con miras a mejorar la consistencia y armonización de la evaluación económica en el ámbito del uso de alcohol. El valor añadido de esta nueva revisión sobre evaluaciones económicas relacionadas con el alcohol es la inclusión de programas que diferencian entre tratamientos para personas con trastornos por uso de alcohol, tratamientos para personas en riesgo de problemas relacionados con el alcohol e intervenciones políticas, legislativas y de aplicación. Esto implica mapear la eficiencia de todas las intervenciones disponibles para abordar este problema de salud pública.

Por tanto, el objetivo de este manuscrito es hacer una revisión sistemática de la literatura de evaluaciones económicas de los tratamientos para las personas con trastornos por uso de alcohol o en riesgo de problemas relacionados con el alcohol y de la legislación de políticas e interven- 
ciones de aplicación, teniendo en cuento los hallazgos de revisiones de la literatura anteriores (Angus, Latimer, Preston, Li y Purshouse, 2014; Barbosa et al., 2010; Chisholm, Doran, Shibuya y Rehm, 2006; Hill, Vale, Hunter, Henderson y Oluboyede, 2017; Hoang et al., 2016; Kaner et al., 2017; Kelly, Abry, Ferri y Humphreys, 2020; Kruse et al., 2020; Ludbrook, 2004; Ludbrook et al., 2002; Mujoomdar y Spry, 2009; Poldrugo, Haeger, Comte, Walburg y Palmer, 2005; Rehm y Barbosa, 2018; Slattery et al., 2002; White, Skirrow, George y Memon, 2018). Los objetivos específicos de esta revisión son: (a) realizar una revisión cualitativa de los aspectos metodológicos de cada uno de los estudios identificados; (b) identificar los programas y las estrategias eficientes más estudiados para tratar a personas con trastornos por uso de alcohol o personas en riesgo de problemas relacionados con el alcohol; y (c) agrupar todas las intervenciones existentes en las tres categorías mencionadas anteriormente $(« A »$ : tratamientos para personas con trastornos por uso de alcohol; «B»: tratamientos para personas en riesgo de problemas relacionados con el alcohol; « $\mathrm{C} »$ : legislación de políticas e intervenciones de aplicación).

\section{Método}

\section{Estrategia de búsqueda}

La revisión sistemática de la literatura se hizo mediante la búsqueda en tres bases de datos: National Health Service Economic Evaluation Database (NHS EED), Health Technology Assessment (HTA), MEDLINE Ovid y PubMed. Todas las bases de datos se buscaron desde su inicio hasta el 24 de julio de 2020, utilizando las palabras clave: (alcohol*:ti or drink*:ti or detoxificat*:ti) cruzado con (cost benefit* or cost effect* or cost utilit* or cost minim* or unit* adj cost or cost*) para las búsquedas en NHS EED y Health Technology Assessment (HTA); y (alcohol\$ [Title] or drink\$[Title] or detoxificat $\$[$ Title $])$ y ( cost\$ benefit $\$$ or cost $\$$ effect $\$$ or cost $\$$ utilit\$ or cost $\$$ minim $\$$ or unit $\$$ adj cost\$) para las búsquedas en MEDLINE y PubMed. Se incluyeron términos de búsqueda en inglés.

\section{Criterios de inclusión}

Los criterios de inclusión consistieron en documentos sobre evaluaciones económicas relacionadas con los seres humanos, sin restricción de tiempo (búsqueda realizada hasta el 24 de julio de 2020), partícipes en programas para el tratamiento de personas con trastornos por uso de alcohol (clasificados como «A» en las tablas de extracción de datos), personas en riesgo de problemas relacionados con el alcohol (clasificadas como «B» en las tablas de extracción de datos) y legislación de políticas e intervenciones de aplicación (clasificadas como «C» en las tablas de extracción de datos). Los manuscritos fueron excluidos si eran artículos de revisión, si no eran evaluaciones económicas completas (análisis comparativo de alternativas de acción en términos tanto de costes [uso de recursos] y de consecuencias [resultados, efectos] con el objetivo de generar medidas de uso incremental de recursos, costes y/o rentabilidad) (Drummond et al., 2015) que proporcionan una ratio de coste-efectividad incremental (RCEI) como resultado, si no utilizaron el término «alcohol» como bebida, o si no se centraron en programas asociados con la reducción o la prevención del consumo de alcohol. Las publicaciones de revisión se guardaron para comprobar la inclusión de los manuscritos identificados en las evaluaciones económicas previas. Las publicaciones incluidas fueron las identificadas a través de la estrategia de búsqueda y algunas otras fueron obtenidas del seguimiento de las citas de los artículos clave identificados.

\section{Método de extracción y síntesis de datos}

Los datos de los artículos incluidos se extrajeron utilizando la misma estructura que la herramienta de extracción de datos estandarizada para evaluaciones económicas en el Joanna Briggs Institute for Evidence Based Practice (JBI-ACTUARI) (The Joanna Briggs Institute, 2014). La calidad y la validez de los estudios incluidos fueron sometidos a doble revisión por dos revisores independientes usando las herramientas estandarizadas de evaluación crítica del Joanna Briggs Institute de JBI-ACTUARI para evaluaciones económicas (The Joanna Briggs Institute, 2014). En caso de discrepancia, ambos revisores revisaron los artículos por segunda vez para llegar a un consenso. No se extrajeron datos adicionales. La revisión sistemática sigue las recomendaciones de los Elementos de información preferidos para revisiones sistemáticas y metaanálisis (PRISMA -P) para informes de revisiones sistemáticas (Moher et al., 2015) ${ }^{1}$.

Los datos extraídos incluirán datos descriptivos sobre (i) la población/participantes del estudio, el nivel de dependencia al alcohol, la intervención, los comparadores y los resultados; (ii) los métodos del estudio, incluido el tipo de diseño de la evaluación, los puntos de vista analíticos; fuente de datos de eficiencia, precios y moneda utilizada para el cálculo de costes, período de análisis; prueba de sensibilidad; medidas de uso de recursos; coste y efecto sobre la salud/efectividad clínica y coste; y (iii) el contexto del estudio (geográfico, año de publicación, atención sanitaria y entorno y cultura de prestación de servicios más amplios). Respecto de la dependencia al alcohol, la décima revisión de la Clasificación internacional de enfermedades y problemas relacionados con la salud (CIE-10) define el síndrome de dependencia como un conjunto de fenómenos

${ }^{1}$ La lista de verificación de PRISMA está disponible bajo petición. 
fisiológicos, conductuales y cognitivos en los que el uso de una sustancia o clase de sustancias adquiere una prioridad mucho más alta para un individuo determinado que otros comportamientos que alguna vez tuvieron mayor valor (Organización Mundial de la Salud, 1992). Este concepto es de mucha importancia en el contexto del alcohol para describir la importancia y el nivel de este comportamiento abusivo y las posibles consecuencias que podría tener. Se resumieron los datos sobre la definición de dependencia al alcohol y de las personas en riesgo de dependencia al alcohol. Todas las intervenciones existentes se clasificaron según el tratamiento para las personas con trastornos por uso de alcohol; tratamientos para personas en riesgo de problemas relacionados con el alcohol; e intervenciones políticas, legislativas y de aplicación. Para ello, se consideraron algunos consejos de los legisladores, las definicio- nes desarrolladas en revisiones publicadas (Barbosa et al., 2010; Chisholm et al., 2006; Ludbrook, 2004; Ludbrook et al., 2002; Slattery et al., 2002) y los resultados obtenidos de esta revisión sistemática de la literatura.

\section{Resultados}

\section{Descripción de los estudios incluidos}

La Figura 1 documenta el diagrama de flujo del proceso que han seguido los artículos durante el estudio y los motivos de exclusión. Fueron incluidas en el análisis 65 evaluaciones económicas especificando 192 estimaciones para el RCEI que cumplieron con los criterios de inclusión iniciales. Fueron analizadas quince revisiones de la literatura (Angus et al., 2014; Barbosa et al., 2010; Chisholm et al., 2006; Hill et al., 2017; Hoang et al., 2016; Kaner et al.,

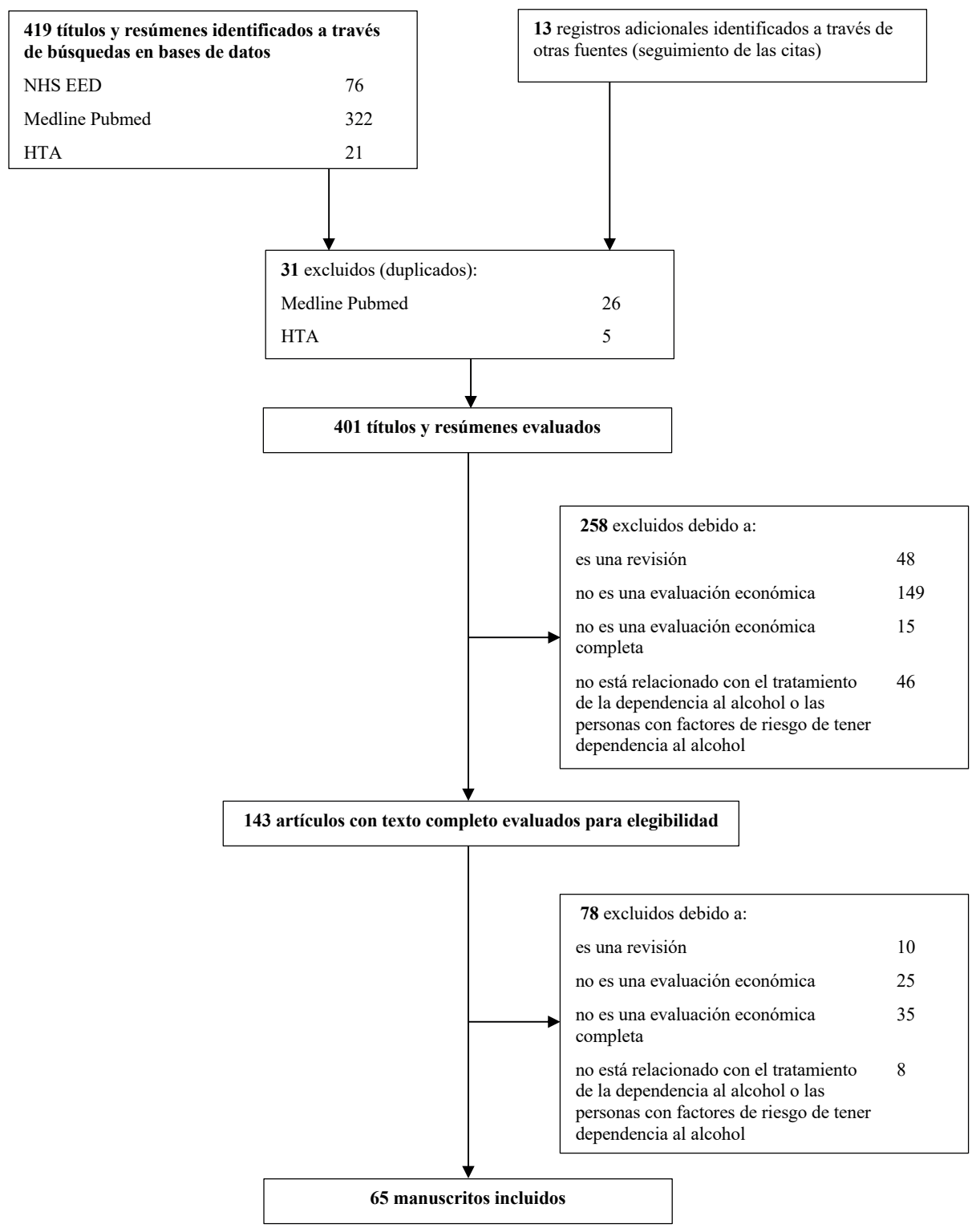

Figura 1. Diagrama de flujo de la identificación y selección del estudio. 
2017; Kelly et al., 2020; Kruse et al. 2020; Ludbrook, 2004; Ludbrook et al., 2002; Mujoomdar y Spry, 2009; Poldrugo et al., 2005; Rehm y Barbosa, 2018; Slattery et al., 2002; White et al., 2018) para comprobar que nuestra revisión incluyó todos los artículos de las mismas. No se incluyeron estudios adicionales porque no eran evaluaciones económicas ni evaluaciones económicas completas (no calcularon la ratio coste-efectividad incremental).

\section{Características de los estudios incluidos}

Todos estos resultados se refieren a los 65 artículos incluidos en la revisión sistemática de la literatura. De estos estudios, hubo uno que evaluó un fármaco (baclofeno) como una intervención en el síndrome de abstinencia de alcohol sin complicaciones. Este estudio también se incluyó en esta revisión sistemática de la literatura porque estas personas todavía tienen trastornos por uso de alcohol o están en ries- go de tener problemas relacionados con el alcohol. De estos 65 artículos, cuatro estudios incluyeron intervenciones clasificadas exclusivamente como prevención primaria, 23 como prevención secundaria (dirigidas a personas con consumo de riesgo de alcohol), 24 como prevención terciaria (dirigidas a personas dependientes al alcohol) y 14 como prevención secundaria y terciaria (dirigidas a personas con consumo de riesgo de alcohol y personas con dependencia al alcohol). Casi el setenta por ciento de los artículos $(\mathrm{n}=$ 44) se publicaron en los últimos diez años (entre 2010 y 2020). Solo el 9,2\% de los artículos $(n=6)$ apareció por primera vez entre 1991 y 2002. El último año de la búsqueda hasta la fecha indicada generó cinco estudios publicados. El Apéndice 1 contiene una lista de los 65 artículos incluidos en la presente revisión sistemática de la literatura. Consulte la Tabla 1 para obtener más detalles sobre las principales características de los estudios incluidos en la Tabla 1.

Tabla 1. Características principales de los estudios incluidos y definiciones de la dependencia al alcohol y personas en riesgo de dependencia al alcohol.

\begin{tabular}{|c|c|c|c|c|c|c|}
\hline Autores & $\begin{array}{l}\text { Tamaño } \\
\text { muestral }\end{array}$ & $\begin{array}{l}\text { Dependencia al } \\
\text { alcohol }^{*}\end{array}$ & $\begin{array}{l}\text { Definición de } \\
\text { dependencia al alcohol }\end{array}$ & Definición de personas en riesgo de dependencia al alcohol & $\begin{array}{l}\text { Tipo de EE } \\
\text { (según los } \\
\text { revisores) }\end{array}$ & Perspectiva* $^{* *}$ \\
\hline Agus A et al., 2019 & 8226 & Sin dependencia & Sin definición incluida & Consumo Episódico Excesivo (CEE) ${ }^{\mathrm{a}}$ & ACE & Financiador \\
\hline Angus C et al., 2014 & ne & - & Sin definición incluida & Sin definición incluida & $\mathrm{ACU}$ & Financiador \\
\hline Barbosa C et al., 2010 & 608 & Dependencia & Sin definición incluida & $\begin{array}{l}\text { Consumo de riesgo de alcohol: } \leq 54,99 \mathrm{~g} / \text { día (mujeres); } \\
\leq 79,99 \mathrm{~g} / \text { día (hombres); consumo nocivo de alcohol: } \leq 55 \mathrm{~g} / \\
\text { día (mujeres); } \leq 80 \mathrm{~g} / \text { día (hombres) }\end{array}$ & $\mathrm{ACU}$ & Financiador \\
\hline Barbosa C et al., 2015 & 9835 & ne & Sin definición incluida & Sin definición incluida & ACE; ACU & Proveedor; Social \\
\hline Barbosa C et al., 2017 & 976 & ne & Sin definición & Sin definición incluida & ACE & Proveedor \\
\hline Barrett B et al., 2006 & 290 & Dependencia & Sin definición & $\begin{array}{l}\text { Un hombre que bebe más de } 8 \text { unidades de alcohol en una } \\
\text { misma ocasión al menos una vez por semana; una mujer que } \\
\text { bebe más de } 6 \text { unidades de alcohol en una misma ocasión al } \\
\text { menos una vez por semana }\end{array}$ & ACE & Social \\
\hline Blankers M et al., 2012 & 136 & Dependencia & $\begin{array}{l}\text { Puntuación en el } \\
\text { AUDIT }>8 \text { y consumo } \\
\text { semanal de más de } 14 \\
\text { unidades de bebida } \\
\text { estándar ( } 10 \mathrm{~g} \text { etanol) }\end{array}$ & Sin definición & ACE; ACU & Social \\
\hline $\begin{array}{l}\text { Brodtkorb T-H et al., } \\
2016\end{array}$ & 100000 & Dependencia & Sin definición & $\begin{array}{l}\text { Hombres/Mujeres: } \\
\text { Riesgo muy alto ( } 1101 />60 \mathrm{~g} \text { por día) } \\
\text { Riesgo alto (61-100/41-60 g por día) } \\
\text { Riesgo medio (41-60/21-40 g por día) } \\
\text { Riesgo bajo (1-40/1-20 g por día) }\end{array}$ & $\mathrm{ACU}$ & Financiador; Social \\
\hline Byrnes JM et al., 2010 & ne & Sin dependencia & Sin definición & Sin definición incluida & $\mathrm{ACU}$ & Financiador \\
\hline Chisholm D et al., 2004 & ne & Dependencia & Sin definición incluida & $\begin{array}{l}\text { El consumo de riesgo y nocivo de alcohol se definió como la } \\
\text { ingesta media de más de } 20 \text { y } 40 \mathrm{~g} \text { de alcohol puro por día } \\
\text { para mujeres y hombres, respectivamente. }\end{array}$ & $\mathrm{ACU}$ & Social \\
\hline Chisholm D et al., 2018 & 529 & ne & Sin definición incluida & $\begin{array}{l}\text { El consumo de riesgo y nocivo de alcohol se definió como la } \\
\text { ingesta media de más de } 20 \text { y } 40 \mathrm{~g} \text { de alcohol puro por día } \\
\text { para mujeres y hombres, respectivamente. }\end{array}$ & $\mathrm{ACU}$ & ne \\
\hline Cobiac L et al., 2009 & ne & Dependencia & Sin definición incluida & Sin definición incluida & $\mathrm{ACU}$ & Financiador \\
\hline $\begin{array}{l}\text { Cordovilla-Guardia S et } \\
\text { al., } 2020\end{array}$ & 294 & Sin dependencia & Sin definición incluida & Sin definición incluida & $A C B$ & Financiador \\
\hline Coulton S et al., 2017 & 529 & Sin dependencia & Sin definición incluida & $\begin{array}{l}\text { Una puntuación en el AUDIT >8 es indicativa de uso de riesgo } \\
\text { de alcohol }\end{array}$ & $\mathrm{ACU}$ & Financiador; Social \\
\hline Cowell Al et al., 2012 & 656 & Dependencia & Sin definición incluida & Al menos un episodio de consumo excesivo de alcohol $^{\mathrm{b}}$ & ACE & Proveedor \\
\hline $\begin{array}{l}\text { Crawford MJ et al., } \\
2015\end{array}$ & 797 & ne & Sin definición incluida & $\begin{array}{l}\text { Hombres que beben más de ocho bebidas estándar en una } \\
\text { ocasión una vez o más al mes, y mujeres que beben más de } \\
\text { seis bebidas estándar en una ocasión una vez o más al mes } \\
\text { (Modified-Single Alcohol Screening Questionnaire [M-SASQ]) }\end{array}$ & ACU & Financiador \\
\hline
\end{tabular}


Evaluaciones económicas de intervenciones dirigidas a la prevención, tratamiento y/o rehabilitación de trastornos por consumo de alcohol: una revisión sistemática

Tabla 1. (cont.).

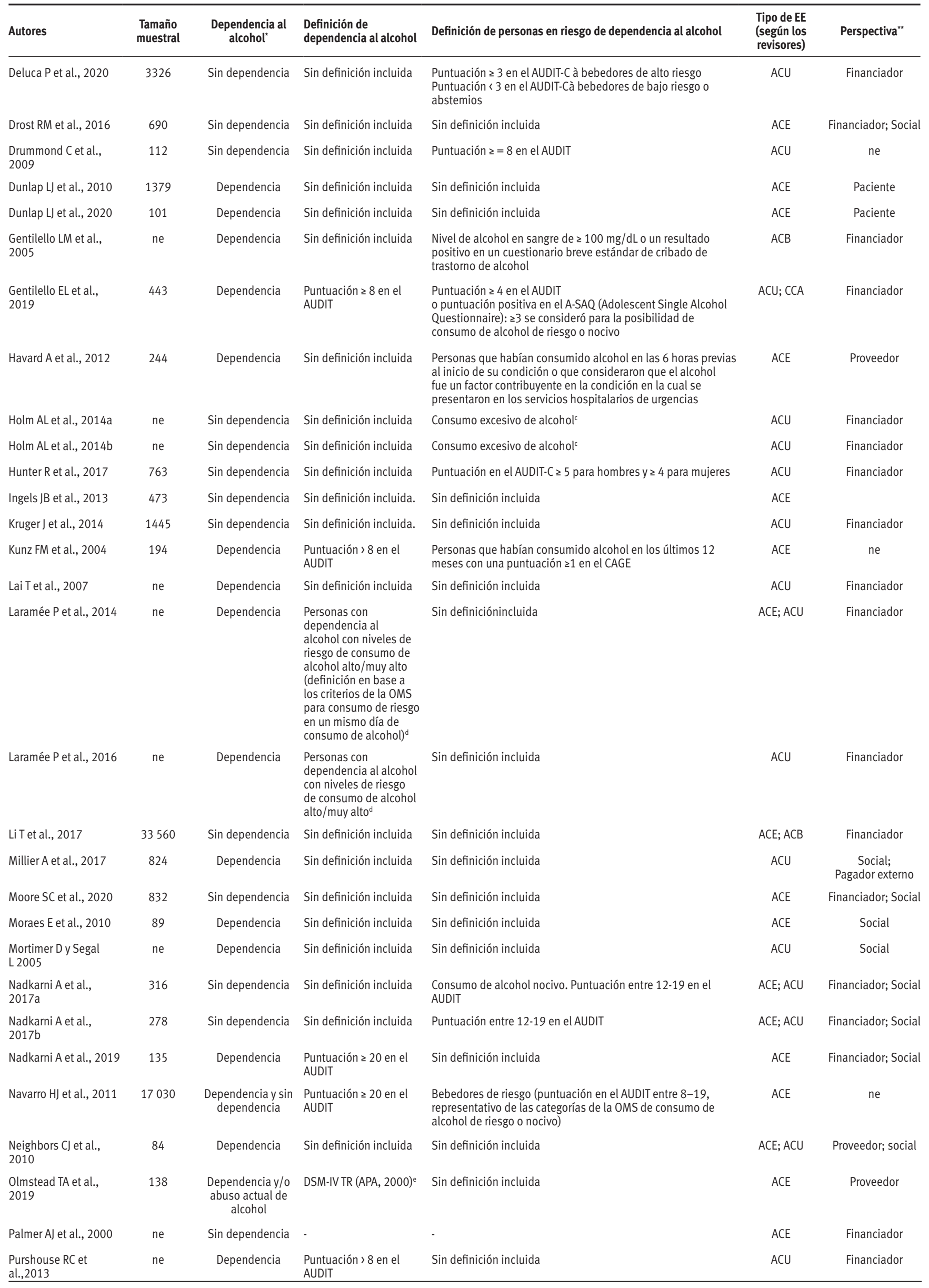


Marta Trapero-Bertran, Dolors Gil-Doménech, Ana Magdalena Vargas-Martínez

Tabla 1. (cont.).

\begin{tabular}{|c|c|c|c|c|c|c|}
\hline Autores & $\begin{array}{l}\text { Tamaño } \\
\text { muestral }\end{array}$ & $\begin{array}{l}\text { Dependencia al } \\
\text { alcohol }^{*}\end{array}$ & $\begin{array}{l}\text { Definición de } \\
\text { dependencia al alcohol }\end{array}$ & Definición de personas en riesgo de dependencia al alcohol & $\begin{array}{l}\text { Tipo de EE } \\
\text { (según los } \\
\text { revisores) }\end{array}$ & Perspectiva** $^{* *}$ \\
\hline Robinson E et al., 2020 & ne & Sin dependencia & Sin definición incluida & Sin definición incluida & ACE & Social \\
\hline Reddy VK et al., 2014 & 60 & Dependencia & Sin definición incluida & Sin definición incluida & ACE & $\begin{array}{l}\text { Financiador; } \\
\text { paciente }\end{array}$ \\
\hline Rychlik R et al., 2003 & 814 & Dependencia & Sin definición incluida & Sin definición incluida & ACE & ne \\
\hline $\begin{array}{l}\text { Schädlich PK y Brecht } \\
\text { JG, } 1998\end{array}$ & 2000 & Dependencia & $\begin{array}{l}\text { Personas que } \\
\text { cumplieron al menos } \\
5 \text { de los criterios del } \\
\text { DSM para dependencia } \\
\text { al alcohol y que eran } \\
\text { dependientes al } \\
\text { alcohol según el Test de } \\
\text { alcoholismo de Munich }\end{array}$ & Sin definición incluida & ACE & Financiador \\
\hline Schulz DN et al., 2014 & 1733 & Sin dependencia & Sin definición incluida & Sin definición incluida & ACE; ACU & Financiador; Social \\
\hline Slattery J et al., 2002 & 1000 & Dependencia & $\begin{array}{l}\text { Definición de } \\
\text { dependencia al } \\
\text { alcohol basada en las } \\
\text { categorías diagnósticas } \\
\text { de la Clasificación } \\
\text { internacional de } \\
\text { enfermedades y } \\
\text { problemas relacionados } \\
\text { con la salud }(\mathrm{CIE}-10)^{f}\end{array}$ & Sin definición incluida & ACE & Financiador \\
\hline Sluiter RL et al., 2018 & ne & Dependencia & Sin definición incluida & Sin definición incluida & ACE & Social \\
\hline Smit F et al., 2011 & 1254000 & Dependencia & $\begin{array}{l}\text { Dependencia al alcohol } \\
\text { basada en los criterios } \\
\text { de la OMS }\end{array}$ & Sin definición incluida & ACB; ACU & Financiador \\
\hline Solberg LI et al., 2008 & 4000000 & Sin dependencia & - & - & $\mathrm{ACU}$ & Financiador; social \\
\hline Sumnall H et al., 2017 & 12738 & Sin dependencia & Sin definición incluida & Consumo Episódico Excesivo (CEE)a & ACE & Social \\
\hline Tariq L et al., 2009 & 1110000 & Dependencia & $\begin{array}{l}\text { Dependencia al alcohol } \\
\text { basada en los criterios } \\
\text { del DSM-IV }\end{array}$ & $\begin{array}{l}\text { «Los grupos de alto riesgo se definen como mujeres que beben } \\
2 \text { o más bebidas estándar de alcohol (i.e., } 20 \mathrm{~g} \text { de etanol) } \\
\text { por día; y hombres que beben } 4 \text { o más bebidas estándar } \\
\text { de alcohol (i.e., } 40 \mathrm{~g} \text { de etanol) por día; sin cumplir con los } \\
\text { criterios del DSM-IV para dependencia al alcohol» }\end{array}$ & ACE; ACU & Financiador \\
\hline $\begin{array}{l}\text { UKATT Research Team, } \\
2005\end{array}$ & 608 & Dependencia & Sin definición incluida & Sin definición incluida & ACU & ne \\
\hline $\begin{array}{l}\text { van den Berg M et al., } \\
2008\end{array}$ & ne & Sin dependencia & Sin definición incluida & $\begin{array}{l}\text { Niveles de consumo de riesgo de alcohol basados en el Modelo } \\
\text { ambiental de enfermedades crónicas }\end{array}$ & ACE; ACU & Financiador \\
\hline Watson J et al., 2013 & 422 & Dependencia & $\begin{array}{l}\text { Puntuación } \geq 20 \text { en el } \\
\text { AUDIT }\end{array}$ & Puntuación $\geq 8$ en el AUDIT & ACU & Financiador \\
\hline Weisner C et al., 2000 & 541 & Dependencia & $\begin{array}{l}\text { Dependencia al alcohol } \\
\text { basada en los criterios } \\
\text { del DSM-IVe }\end{array}$ & Sin definición incluida & ACE & ne \\
\hline Wutzke SE et al., 2001 & ne & Sin dependencia & Sin definición incluida & $\begin{array}{l}\text { Clasificación de consumo de alcohol según los criterios del } \\
\text { Consejo Nacional de Investigación Médica y de Salud de } \\
\text { Australiai }\end{array}$ & ACE & Financiador \\
\hline Zarkin GA et al., 2008 & 1383 & Dependencia & $\begin{array}{l}\text { Dependencia al alcohol } \\
\text { basada en los criterios } \\
\text { del DSM-IVe }\end{array}$ & Sin definición incluida & ACE & Proveedor \\
\hline
\end{tabular}

Nota. ne: información no especificada; ACE: análisis de coste-efectividad; ACU: análisis de coste-utilidad; DSM: Manual Diagnóstico y Estadístico de los Trastornos Mentales

* Definición de dependencia como un conjunto de fenómenos fisiológicos, conductuales y cognitivos en los que el uso de una sustancia o clase de sustancias adquiere una prioridad mucho más alta para un individuo determinado que otros comportamientos que alguna vez tuvieron mayor valor (Organización Mundial de la Salud, 1992).

**Perspectiva del financiador: consideración de los resultados y los costes de interés para la entidad que financia la intervención; Perspectiva social: recolección de todos los resultados relevantes y los costes asumidos por los proveedores y los beneficiarios potenciales (la sociedad en general); Perspectiva del proveedor: consideración de los resultados y los costes de interés para la entidad que implementa la intervención (i.e., Sistema Nacional de Salud, universidades, etc.); Perspectiva del paciente: consideración de los resultados y los costes de interés para el paciente.

a Consumo Episódico Excesivo (CEE): definido como el consumo de 6 o más unidades en una misma ocasión para estudiantes masculinos y de 4,5 o más unidades para estudiantes femeninas. ${ }^{b}$ Episodio de consumo excesivo de alcohol: un hombre que bebe 50 más bebidas en una misma ocasión o una mujer que bebe 40 más bebidas en una misma ocasión.

'Consumo excesivo de alcohol: Consumo de riesgo de alcoholà12-23,9 g/día para mujeres y 24-35,9 g/día para hombres. Consumo nocivo de alcoholà > $24 \mathrm{~g} /$ día para mujeres y > $36 \mathrm{~g} /$ día para hombres. ${ }^{d}$ Personas con dependencia al alcohol con niveles de riesgo de consumo de alcohol alto/muy alto según los criterios de la OMS para consumo de riesgo en un mismo día de consumo de alcohol: $\leq 41 \mathrm{~g} /$ día para mujeres; $\leq 61 \mathrm{~g} /$ día para hombres. ${ }^{e}$ Definición de dependencia al alcohol según los criterios del DSM-IV TR (APA, 2000). Referencia: American Psychiatric Association (APA). (2000). DSM-IV-TR. Barcelona: Masson. ${ }^{f}$ Definición dependencia al alcohol según la CIE-10. Referencia: Organización Mundial de la Salud. CIE-10: Clasificación internacional de enfermedades y problemas relacionados con la salud (décima edición, vol. 1) Ginebra, Suiza. 1992. ${ }^{8}$ Definición de dependencia al alcohol, según los criterios de la OMS, como el cumplimiento de «un mínimo de tres de los siguientes criterios: tolerancia; síntomas de abstinencia; deterioro del control; obsesión con la compra y/o el consumo; deseo persistente 0 esfuerzos fallidos para dejarlo; deterior continuo a nivel social, laboral o de ocio; y continuación del uso a pesar de las consecuencias adversas». ${ }^{\mathrm{h}}$ Definiciones de las categorías de consumo de alcohol: moderado: menos de dos bebidas estándar ( $20 \mathrm{~g})$ por día para mujeres, y menos de cuatro bebidas estándar ( $40 \mathrm{~g})$ por día para hombres; excesivo: $2-4$ bebidas estándar (20-40 g) por día para mujeres, y 4-6 bebidas estándar (40-60 g) por día para hombres; nociva: más de 4 bebidas estándar ( $>40 \mathrm{~g})$ por día para mujeres, y más de 6 bebidas estándar ( $>$ 60 g) por día para hombres. 'Clasificación de consumo de alcohol según los criterios del Consejo Nacional de Investigación Médica y de Salud de Australia (NHMRC) (NHMRC, 1992). Según estos criterios, el consumo seguro es menos de $40 \mathrm{~g}$ (cuatro bebidas estándar) para hombres y menos de $20 \mathrm{~g}$ (dos bebidas estándar) para mujeres, con dos días a la semana sin consumo de alcohol. Niveles de consumo de alcohol por encima de estos niveles se considera «de riesgo» y por encima de $60 \mathrm{~g}$ y $40 \mathrm{~g}$ al día, respectivamente, como «nocivo». 
De los 65 artículos incluidos, el 23,1\% $(\mathrm{n}=15)$ era de Europa (uno de España), el 21,5 \% ( $\mathrm{n}=14)$ de los Estados Unidos, el 12,3\% ( $\mathrm{n}=8)$ de Australia, el 26,2 \% ( $\mathrm{n}=17)$ del Reino Unido, el 6,2\% $(\mathrm{n}=4)$ de India y el restante de Brasil, Estonia e Italia. Cuatro de los artículos estudiaron más de un país. La edad promedia de las poblaciones, ponderada por muestra, incluidas en el estudio fue de aproximadamente 38 años, aunque solo el $46 \%$ de los estudios informó la edad. La mayoría de los estudios (73,8 \%; $\mathrm{n}=48$ ) informó el tamaño muestral; 18 comprendió una muestra superior a 10 000; sin embargo, tres comprendió una muestra inferior a 100 personas. El género de las personas se especificó en el 58,7 \% de los estudios incluidos en la evaluación económica; de esos, el 35,1 \% era solo hombres. Solo un estudio especificó el nivel socioeconómico de los participantes. Solo dos estudios $(3,2 \%)$ ofrecieron una compensación monetaria por la participación en el estudio. Treinta y dos $(50,8 \%)$ estudios se basaron en ensayos, todos aleatorios con la excepción de dos estudios.

Más de la mitad de los estudios $(56,9 \% ; n=37)$ declaró que los participantes tenían dependencia al alcohol. Sin embargo, solo diecisiete $(47,2 \%)$ definieron esta condición, cuya definición se basó en gramos por día o semana, o unidades/bebidas consumidas por día/semana, o en base a la puntuación en el AUDIT, los criterios de la OMS, la CIE-10, o en base a la entrevista diagnóstica para la dependencia de sustancias psicoactivas (DSM). Un elevado número de estudios $(\mathrm{n}=25 ; 39,7 \%)$ afirmó que sus participantes atribuían condiciones de salud al alcohol, entre ellas, enfermedades cardiovasculares $(\mathrm{n}=19)$ y hepáticas $(\mathrm{n}=14)$ y cáncer $(\mathrm{n}=$ 14) como las más frecuentes, seguidas de accidentes de tráfico $(n=11)$. Consulte la Tabla 1 para obtener más detalles.

\section{Evaluación de la calidad metodológica de los estudios incluidos}

Se utilizó el JBI-ACTUARI para evaluar la calidad de los estudios incluidos. Todos los estudios alcanzaron un nivel de calidad aceptable para su inclusión en la revisión sistemática. Comúnmente, estos resultados se refieren a los 192 RCEI generados por la revisión sistemática de la literatura. A continuación se presenta la información en relación a los ítems medidos a través del JBI-ACTUARI relacionados con una pregunta/objetivo bien definido, descripción integral de alternativas, identificación de costes y resultados relevantes para cada alternativa y su ajuste por tiempo diferencial, efectividad clínica, análisis incremental de costes y consecuencias, y análisis de sensibilidad. En general, todos los artículos definieron adecuadamente la pregunta de investigación y los programas o intervenciones comparados en el análisis. La descripción de alternativas fue amplia, aunque en muchas ocasiones (49,2 \%), los artículos no informaron el tamaño muestral de cada brazo y la duración de la intervención. El $50 \%$ de los artículos tomó la perspectiva del financiador sanitario, principalmente el Sistema Nacional de Salud, y solo el 18,8 \% adoptó una perspectiva social. El horizonte de vida se utilizó en el 57,1 \% de los estudios, con poca frecuencia de un horizonte temporal inferior a un año. Los costes y los resultados se ajustaron por tiempo diferencial en más del $50 \%$ de los artículos, mientras que el 39,68 \% presentó los resultados y costes sin un ajuste apropiado. Se estableció la efectividad clínica, utilizando principalmente una medida de calidad de vida (47,6 \%) seguida de una medida clínica $(39,7 \%)$ o ambos a la vez $(12,7 \%)$. Todos los artículos explican cómo han obtenido la estimación de la eficiencia. El $46 \%$ de los papeles no especificó qué tipos de costes incluyó su análisis. De los que especificaron los tipos de costes, el 79,4 1\% de los artículos incluyó los costes directos relacionados con la atención sanitaria, así como diferentes tipos de costes, como los costes directos no relacionados con la salud, los costes de los pacientes o los costes de intervención. Solo diez artículos $(26,5 \%)$ incluyeron las pérdidas de productividad. Tres artículos incluyeron los costes de atención informal. De todos los artículos que especifican el uso de la perspectiva social, el 81,8 \% de los mismos incluyó costes relacionados con la pérdida de productividad. Los costes y los resultados se han medido con precisión, aunque en muchos casos el uso de los recursos no se informó por separado. Es importante en cuanto a recursos separar los costes/precios por razones de transparencia, comparabilidad y transferibilidad. Para comprender y evaluar si los datos de costes utilizados en la evaluación económica eran razonables, siempre es más fácil si los recursos se informan por separado de los costes/precios. Casi el $60 \%$ de los estudios $(n=35)$ hizo un análisis de coste-utilidad. El $49 \%$ de los artículos utilizó un análisis de decisiones para estimar los costes y los resultados, el 48,4\% de ellos son modelos diferentes de un árbol de decisión y un modelo de Márkov. El 88,9 \% de los artículos realizó un análisis de sensibilidad, en su mayoría determinístico $(57,1 \%)$ en lugar de probabilístico $(42,9 \%)$. De los que realizan un análisis de sensibilidad probabilístico, la gran mayoría $(79,2 \%)$ representó una curva de aceptabilidad de coste-efectividad.

\section{Hallazgos de la revisión}

Estos resultados se refieren a los 192 RCEI generados por la revisión sistemática de la literatura. En resumen, tanto para personas con dependencia al alcohol como personas con factores de alto riesgo de hacerse dependientes al alcohol, las intervenciones psicológicas fueron las más evaluadas en términos de eficiencia; demostraron ser rentables o dominantes en comparación con la ausencia de intervención. Para las intervenciones farmacológicas dirigidas a personas con dependencia al alcohol, los fármacos más utilizados y evaluados fueron nalmefeno y acamprosato, seguido de otros opioides y antagonistas opiáceos y otros fármacos como baclofeno y disfulfiram. Las intervenciones políticas, legislativas y de aplicación se basaron principalmente en subidas de impuestos, observando una 
tendencia para la dominancia o la rentabilidad en comparación con la ausencia de intervención.

Las diferentes evaluaciones económicas halladas abarcan una gama de intervenciones, tales como intervenciones psicosociales; tratamientos farmacológicos; intervenciones breves; e intervenciones políticas, legislativas y de aplicación. También se incluyeron otras intervenciones, como tratamiento residencial, pruebas aleatorias de alcoholemia, telemarketing de médicos de cabecera, etc. Consulte la Tabla 2 para conocer las definiciones de los diversos programas e intervenciones informados por esta revisión sistemática de la literatura y otras cuatro revisiones publicadas anteriormente (Barbosa et al., 2010; Chisholm et al., 2006; Ludbrook et al., 2002; Slattery et al., 2002) que ya realizaban homogeneizaban la taxonomía para el tratamiento de personas con trastornos por uso de alcohol o personas en riesgo de tener problemas relacionados con el alcohol.

Todas estas diferentes intervenciones se han clasificado según la disponibilidad de evidencia sobre eficiencia de acuerdo con los objetivos de los programas. La clasificación utilizada fue también basada en las revisiones publicadas anteriormente (Barbosa et al., 2010; Chisholm et al., 2006; Ludbrook et al., 2002; Slattery et al., 2002), juntamente con esta revisión sistemática. La Tabla S1 de material suplementario muestra la definición de estas intervenciones y la Tabla 2 muestra la clasificación de los tipos de programas de tratamiento para la dependencia al alcohol.

Tabla 2. Tipos de programas de tratamiento para la dependencia al alcohol (Fuente: propia; y Barbosa et al., 2010; Chisholm et al., 2004; Ludbrook et al., 2002; Slattery et al., 2002).

\begin{tabular}{ll}
\hline & A. Tratamientos para personas con dependencia al alcohol \\
\hline Intervenciones psicosociales & Intervenciones farmacológicas \\
Entrevista motivacional & Acamprosato \\
Terapia de estímulo de la motivación & Opioides y antagonistas opiáceos (i.e., naltrexona, nalmefeno, etc.) \\
Terapia de habilidades y relaciones sociales & Disulfiram \\
Entrenamiento en autocontrol conductual & Litio \\
Entrenamiento en habilidades sociales/de afrontamiento & Inhibidores selectivos de la recaptación de serotonina \\
Terapia matrimonial, de pareja o de familia & Benzodiazepinas \\
Exposición de señales orientada a la moderación & Baclofeno \\
Prevención de la recaída & Betabloqueadores \\
Intervención a largo plazo & Agonistas adrenérgicos de los receptores alfa y beta \\
Terapia cognitivo-conductual & \\
Terapia de facilitación de 12 pasos & Otras intervenciones (i.e., tratamiento residencial) \\
Terapia grupal & Intervenciones combinadas \\
Enfoque de refuerzo comunitario & \\
\hline
\end{tabular}

B. Tratamientos para personas en riesgo de problemas relacionados con el alcohol

Intervenciones breves

Intervenciones escolares

Programa de intervención en habilidades familiares

Otras intervenciones (i.e., telemarketing de médicos de cabecera, eHealth)

Intervenciones combinadas

SBIRT (cribado, intervención breve y derivación a tratamiento)

Atención escalonada

AMPP (Programa de prevención de abuso de alcohol)

AIMS (Tratamiento de desintoxicación de alcohol)

Iniciativas de prevención comunitaria

C. Intervenciones políticas, legislativas y de aplicación

Legislación/aplicación de la conducción bajo los efectos del alcohol

(pruebas de alcoholemia aleatorias)

Control/prohibición de la publicidad

Subida de impuestos

Control de licencias

Edad legal para beber

Campañas en los medios de comunicación

Intervenciones combinadas

Formación para los bármanes

Nota. *Aparecen sombreadas las intervenciones para las cuales los autores han hallado evidencia de eficiencia.

Los tratamientos para personas con dependencia al alcohol $(32,8 \%$; $=63)$ han sido los más evaluados en comparación con los tratamientos para personas en riesgo de tener problemas relacionados con el alcohol $(28,6 \% ; \mathrm{n}=$ 55) y las intervenciones políticas, legislativas y de aplicación $(22,9 \% ; n=44)$. Estos porcentajes se han calculado de acuerdo con el número total de comparaciones halladas en términos de eficiencia $(n=192)$. Además, el restante $15,3 \%$ de las comparaciones evaluó una combinación de diferentes tipos de intervenciones $(n=29)$. Consulte la Tabla 3 para obtener más detalles. 
Evaluaciones económicas de intervenciones dirigidas a la prevención, tratamiento y/o rehabilitación de trastornos por consumo de alcohol: una revisión sistemática

Tabla 3. Comparación de los tipos de intervención (65 publicaciones; 192 comparaciones en términos de eficiencia).

\begin{tabular}{|c|c|c|c|}
\hline Intervención & $\begin{array}{l}\text { Comparador (véase la Tabla } 2 \text { para definiciones } \\
\text { generales de estas intervenciones) }\end{array}$ & $\begin{array}{l}\text { Resultados en términos de } \\
\text { eficiencia* }\end{array}$ & Referencias \\
\hline \multicolumn{4}{|c|}{ A: Tratamientos para personas con dependencia al alcohol $(n=63)$} \\
\hline \multicolumn{4}{|l|}{ Intervenciones psicosociales $(n=18)$} \\
\hline \multirow{3}{*}{$\begin{array}{l}\text { Entrevista motivacional } \\
(n=3)\end{array}$} & Entrevista motivacional (> 3 meses) $(n=1)$ & Dominado & Cowell AJ et al., 2012 \\
\hline & Sin intervención $(n=1)$ & Dominante & Slattery J et al., 2002 \\
\hline & Intervención breve $(\mathrm{n}=1)$ & Rentable & Neighbors CJ et al., 2010 \\
\hline Terapia de estímulo de la motivación $(n=1)$ & Intervenciones breves $(\mathrm{n}=1)$ & Rentable & Mortimer D y Segal L, 2005 \\
\hline Terapia de habilidades y relaciones sociales $(n=2)$ & Terapia de estímulo de la motivación $(n=2)$ & $\begin{array}{c}\text { Indiferente } \\
\text { (ambos rentables) }(n=1) \\
\text { Rentable }(n=1)\end{array}$ & $\begin{array}{l}\text { Barbosa C et al., 2005; UKATT } \\
\text { Research Team, } 2005\end{array}$ \\
\hline Entrenamiento en autocontrol conductual $(n=1)$ & Sin intervención $(n=1)$ & Dominancia & Slattery J et al., 2002 \\
\hline $\begin{array}{l}\text { Entrenamiento en habilidades sociales/de } \\
\text { afrontamiento }(n=1)\end{array}$ & Sin intervención $(n=1)$ & Dominante & Slattery J et al., 2002 \\
\hline Terapia matrimonial, de pareja o de familia $(n=1)$ & Sin intervención $(n=1)$ & Dominante & Slattery J et al., 2002 \\
\hline $\begin{array}{l}\text { Exposición de señales orientada a la moderación } \\
(\mathrm{n}=1)\end{array}$ & Entrenamiento en autocontrol conductual $(n=1)$ & Rentable & Mortimer D, Segal L, 2005 \\
\hline Prevención de la recaída $(n=1)$ & Sin intervención $(n=1)$ & ne & Slattery J et al., 2002 \\
\hline $\begin{array}{l}\text { Intervención a largo plazo (consulta para los recursos } \\
\text { humanos entrenados) }(n=1)\end{array}$ & Intervenciones breves $(\mathrm{n}=1)$ & No rentable & Holm AL et al., $2014 a$ \\
\hline Intervención de eHealth $(n=1)$ & Práctica actual (a) (n=1) & Rentable & Smit F et al., 2011 \\
\hline Intervención conductual combinada ( $\mathrm{n}=2)$ & Tratamiento médico + placebo $(n=2)$ & Dominado & $\begin{array}{l}\text { Dunlap LJ et al., 2010; Zarkin } \\
\text { GA et al., } 2008\end{array}$ \\
\hline $\begin{array}{l}\text { Intervenciones breves psicosociales } \\
\text { ( } 3 \text { visitas) para personas } \\
\text { con uso de alcohol de riesgo y nocivo } \\
\text { (cobertura del } 50 \%) .(n=2)\end{array}$ & Sin intervención o Situación actual $(n=2)$ & Rentable & Chisholm D et al., 2018 \\
\hline $\begin{array}{l}\text { Atención habitual mejorada (EUC) + Terapia para } \\
\text { problemas relacionados con el alcohol (CAP) }(n=1)\end{array}$ & EUC solo $(n=1)$ & Rentable & Nadkarni et al., 2019 \\
\hline \multicolumn{4}{|l|}{ Intervenciones farmacológicas $(n=7)$} \\
\hline \multirow{2}{*}{ Acamprosato $(n=4)$} & Placebo $(n=3)$ & Dominante $(n=1)$ & $\begin{array}{l}\text { Rychlik R et al., 2003; Schädlich } \\
\text { PKy Brecht JG, 1998; Slattery J }\end{array}$ \\
\hline & Atención habitual $(n=1)$ & Rentable $(n=1)$ & $\begin{array}{l}\text { et al., 2002; Torfs K y De Graeve } \\
\text { D, } 1991\end{array}$ \\
\hline Baclofeno $(n=1)$ & Benzodiazepinas (clordiazepóxido) $(\mathrm{n}=1)$ & Dominado & Reddy VK et al., 2014 \\
\hline Opioide o antagonista opiáceo $(\mathrm{n}=1)$ & Placebo $(n=1)$ & ne & Slattery J et al., 2002 \\
\hline Disulfiram $(n=1)$ & Placebo $(n=1)$ & ne & Slattery J et al., 2002 \\
\hline \multicolumn{4}{|l|}{ Otras intervenciones $(n=1)$} \\
\hline Tratamiento residencial $(n=1)$ & $\begin{array}{l}\text { Legislación de la conducción bajo los efectos del alcohol } \\
(\mathrm{n}=1)\end{array}$ & No rentable & Cobiac L et al., 2009 \\
\hline \multicolumn{4}{|l|}{ Intervenciones combinadas $(n=37)$} \\
\hline $\begin{array}{l}\text { Entrevista motivacional + visitas a domicilio (al inicio y } \\
\text { fin del tratamiento) }(n=1)\end{array}$ & $\begin{array}{l}\text { Entrevista motivacional + prevención de la recaída } \\
(\mathrm{n}=1)\end{array}$ & Rentable & Moraes E et al., 2010 \\
\hline $\begin{array}{l}\text { Entrevista motivacional + terapia cognitivo- } \\
\text { conductual + apoyo terapéutico }(\mathrm{n}=1)\end{array}$ & $\begin{array}{l}\text { Entrevista motivacional + terapia cognitivo-conductual } \\
(\mathrm{n}=1)\end{array}$ & Rentable & Blankers M et al., 2012 \\
\hline $\begin{array}{l}\text { Apoyo psicosocial + opioide o antagonista opiáceo } \\
\text { (nalmefeno) }(\mathrm{n}=2)\end{array}$ & Apoyo psicosocial $(n=2)$ & Rentable; Dominante & Laramée P et al., 2014, 2016 \\
\hline \multirow{2}{*}{$\begin{array}{l}\text { Tratamiento médico + intervención conductual } \\
\text { combinada + acamprosato }(n=2)\end{array}$} & Tratamiento médico + placebo $(n=1)$ & Dominado & Dunlap LJ et al., 2010 \\
\hline & $\begin{array}{l}\text { Tratamiento médico + opioide o antagonista opiáceo + } \\
\text { acamprosato }(\mathrm{n}=1)\end{array}$ & Dominado & Zarkin GA et al., 2008 \\
\hline \multirow{2}{*}{$\begin{array}{l}\text { Tratamiento médico + intervención conductual } \\
\text { combinada + placebo }(n=2)\end{array}$} & $\begin{array}{l}\text { Tratamiento médico + opioide o antagonista opiáceo } \\
(\mathrm{n}=1)\end{array}$ & Dominado & Zarkin GA et al., 2008 \\
\hline & Tratamiento médico + placebo $(n=1)$ & Dominado & Dunlap LJ et al., 2010 \\
\hline \multirow{2}{*}{$\begin{array}{l}\text { Tratamiento médico + intervención conductual } \\
\text { combinada + opioide o antagonista opiáceo } \\
(n=2)\end{array}$} & $\begin{array}{l}\text { Tratamiento médico + opioide o antagonista opiáceo + } \\
\text { acamprosato }(n=1)\end{array}$ & Dominado & Zarkin GA et al., 2008 \\
\hline & Tratamiento médico + placebo $(n=1)$ & Dominado & Dunlap LJ et al., 2010 \\
\hline \multirow{2}{*}{$\begin{array}{l}\text { Tratamiento médico + intervención conductual } \\
\text { combinada + opioide o antagonista opiáceo + } \\
\text { acamprosato }(n=2)\end{array}$} & $\begin{array}{l}\text { Tratamiento médico + opioide o antagonista opiáceo + } \\
\text { acamprosato }(\mathrm{n}=1)\end{array}$ & Dominado & Zarkin GA et al., 2008 \\
\hline & Tratamiento médico + placebo $(n=1)$ & Dominado & Dunlap LJ et al., 2010 \\
\hline
\end{tabular}


Tabla 3. (cont.).

\begin{tabular}{|c|c|c|c|}
\hline Intervención & $\begin{array}{l}\text { Comparador (véase la Tabla } 2 \text { para definiciones } \\
\text { generales de estas intervenciones) }\end{array}$ & $\begin{array}{l}\text { Resultados en términos de } \\
\text { eficiencia* }\end{array}$ & Referencias \\
\hline \multirow{2}{*}{$\begin{array}{l}\text { Tratamiento médico + opioide o antagonista opiáceo + } \\
\text { acamprosato }(n=2)\end{array}$} & Tratamiento médico + placebo $(n=1)$ & Rentable & Dunlap LJ et al., 2010 \\
\hline & $\begin{array}{l}\text { Tratamiento médico + opioide o antagonista opiáceo } \\
(\mathrm{n}=1)\end{array}$ & Rentable & Zarkin GA et al., 2008 \\
\hline $\begin{array}{l}\text { Tratamiento médico + opioide o antagonista opiáceo } \\
(\mathrm{n}=2)\end{array}$ & Tratamiento médico + placebo $(n=2)$ & Rentable & $\begin{array}{l}\text { Dunlap LJ et al., 2010; Zarkin } \\
\text { GA et al., } 2008\end{array}$ \\
\hline \multirow[b]{2}{*}{ Tratamiento médico + acamprosato $(n=2)$} & Tratamiento médico + placebo $(n=1)$ & Dominado & Dunlap LJ et al., 2010 \\
\hline & $\begin{array}{l}\text { Tratamiento médico + opioide o antagonista opiáceo } \\
(\mathrm{n}=1)\end{array}$ & Dominado & Zarkin GA et al., 2008 \\
\hline $\begin{array}{l}\text { Opioide } 0 \text { antagonista opiáceo + tratamiento } \\
\text { residencial }(\mathrm{n}=1)\end{array}$ & Pruebas de alcoholemia aleatorias $(n=1)$ & No rentable & Cobiac L et al., 2009 \\
\hline $\begin{array}{l}\text { Terapia grupal de parejas + tratamiento } \\
\text { individual }(\mathrm{n}=8)\end{array}$ & $\begin{array}{l}\text { Terapia estándar de parejas + tratamiento individual } \\
(\mathrm{n}=8)\end{array}$ & Dominante o rentable & Dunlap LJ et al., 2020 \\
\hline \multirow{2}{*}{ Nalmefeno + apoyo psicosocial $(n=10)$} & Apoyo psicosocial solo $(\mathrm{n}=2)$ & Rentable o dominante & \multirow{2}{*}{$\begin{array}{l}\text { Brodtkorb T-H et al., 2016; } \\
\text { Millier A et al., } 2017\end{array}$} \\
\hline & Sin tratamiento $(n=4)$ & Rentable o dominante & \\
\hline $\begin{array}{l}\text { Prueba de detección de OPRM1: Asignación a } \\
\text { tratamiento con naltrexona para pacientes con TUA } \\
\text { portadores del alelo } \mathrm{G} \text { en el gen OPRM1 }(\mathrm{n}=1)\end{array}$ & $\begin{array}{l}\text { Sin prueba de detección de OPRM1: Asignación } \\
\text { aleatorizado (sin genotipado) a tratamiento } \\
\text { farmacológico con naltrexona o acamprosato }(n=1)\end{array}$ & Rentable & Sluiter et al., 2018 \\
\hline \multicolumn{4}{|c|}{ B: Tratamientos para personas en riesgo de problemas relacionados con el alcohol $(n=55)$} \\
\hline \multicolumn{4}{|l|}{ Intervenciones breves $(n=26)$} \\
\hline \multirow{5}{*}{$\begin{array}{l}\text { Intervenciones breves (presencial) } \\
(\mathrm{n}=19)\end{array}$} & Intervención breve (> 3 meses) $(n=1)$ & Dominado & Cowell AJ et al., 2012 \\
\hline & Pruebas de alcoholemia aleatorias $(n=1)$ & Rentable & Cobiac L et al., 2009 \\
\hline & Sin intervención $(n=15)$ & $\begin{array}{l}\text { Dominante }(n=2) \\
\text { Dominante o rentable } \\
\quad(n=3) \\
\text { Rentable }(n=10)\end{array}$ & $\begin{array}{l}\text { Angus C et al., 2014; Chisholm } \\
\text { D et al., } 2004(n=3) ; \text { Gentilello } \\
\text { LM et al., } 2005 ; \text { Lai T et al., } \\
2007 ; \text { Mortimer D y Segal L, } \\
2005(n=2) ; \text { Purshouse RC } \\
\text { et al., 2013; Solberg Ll et al., } \\
2008(n=2) ; \text { Tariq L et al., 2009; } \\
\text { Wutzke SE et al., } 2001 \text { (n=2); } \\
\text { Havard et al., } 2012\end{array}$ \\
\hline & Control (b) $(n=1)$ & Rentable & Wutzke SE et al., 2001 \\
\hline & Situación actual (c) (n=1) & Coste-beneficio & $\begin{array}{l}\text { Cordovilla-Guardia S et al., } \\
2020\end{array}$ \\
\hline Intervenciones breves (telefónica) $(\mathrm{n}=1)$ & Intervenciones breves (presencial) $(n=1)$ & Dominante & Holm AL et al., 2014b \\
\hline $\begin{array}{l}\text { Strong African American Families-Teen program } \\
(\text { SAAF-T) }(n=1)\end{array}$ & Entrenamiento de control de la atención (ACI) $(n=1)$ & Rentable & Ingels JB et al., 2013 \\
\hline $\begin{array}{l}\text { Retroalimentación personalizada y asesoramiento } \\
\text { breve (PFBA) (presencial) }(n=2)\end{array}$ & \multirow{2}{*}{ - Grupo de control (solo cribado) $(n=2)$} & $\begin{array}{c}\text { No rentable }(n=2) \text { (en las } \\
\text { pruebas de bajo riesgo y } \\
\text { alto riesgo) } \\
\end{array}$ & \multirow{2}{*}{ Deluca P et al., 2020} \\
\hline $\begin{array}{l}\text { Retroalimentación personalizada + intervención breve } \\
\text { con smartphone o en línea }(e B I)(n=2)\end{array}$ & & $\begin{array}{l}\text { Dominado }(n=2) \text { (en las } \\
\text { pruebas de bajo riesgo y } \\
\text { alto riesgo) }\end{array}$ & \\
\hline $\begin{array}{l}\text { Asesoramiento breve (presencial) + ofrecimiento de } \\
\text { una cita con un profesional especializado en salud y el } \\
\text { alcohol (AHW) }(n=1)\end{array}$ & $\begin{array}{l}\text { Tratamiento de control: folleto informativo de la salud en } \\
\text { general }(n=1)\end{array}$ & No rentable & Crawford MJ et al., 2015 \\
\hline \multicolumn{4}{|l|}{ Otras intervenciones $(n=22)$} \\
\hline $\begin{array}{l}\text { Correo electrónico con un informe de retroalimentación } \\
\text { sobre los patrones personales de uso de alcohol } \\
(n=1)\end{array}$ & $\begin{array}{l}\text { Correo electrónico con un informe de retroalimentación } \\
\text { sobre los patrones personales de uso de alcohol }(>3 \\
\text { meses })(n=1)\end{array}$ & ne & Cowell AJ et al., 2012 \\
\hline Intervención de eHealth $(\mathrm{n}=1)$ & Sin intervención $(n=1)$ & Rentable & Smit F et al., 2011 \\
\hline Escucha activa no directiva $(n=1)$ & $\begin{array}{l}\text { Intervención breve (evaluación y retroalimentación) } \\
(\mathrm{n}=1)\end{array}$ & No rentable & Mortimer D y Segal L, 2005 \\
\hline Programa secuencial en línea de varias sesiones $(n=1)$ & Programa simultáneo en línea de varias sesiones $(n=1)$ & Dominado (ACU) & \multirow{3}{*}{ Schulz DN et al., 2014} \\
\hline Programa secuencial en línea de varias sesiones $(n=1)$ & \multirow{2}{*}{ - Control $(n=1)$} & $\begin{array}{l}\text { Rentable (ACE) } \\
\text { Dominado (ACU) }\end{array}$ & \\
\hline Programa simultáneo en línea de varias sesiones $(n=1)$ & & $\begin{array}{c}\text { Rentable (ACE) } \\
\text { Dominado (ACU) }\end{array}$ & \\
\hline $\begin{array}{l}\text { Intervención en línea de teoría sobre la intervención } \\
\text { conductual para la salud (U@Uni) }(n=1)\end{array}$ & Control $(n=1)$ & Rentable & Kruger J et al., 2014 \\
\hline Tratamiento breve (TB) (servicio SBIRT) $(n=1)$ & Intervención breve (IB) (servicio SBIRT) $(n=1)$ & Rentable & Barbosa C et al., 2017 \\
\hline $\begin{array}{l}\text { Modelo de servicios de tratamiento para la } \\
\text { intoxicación por alcohol (AIMS) }(n=6)\end{array}$ & Atención habitual $(n=6)$ & Dominante o rentable & Moore SC et al., 2020 \\
\hline
\end{tabular}


Evaluaciones económicas de intervenciones dirigidas a la prevención, tratamiento y/o rehabilitación de trastornos por consumo de alcohol: una revisión sistemática

Tabla 3. (cont.).

\begin{tabular}{|c|c|c|c|}
\hline Intervención & $\begin{array}{l}\text { Comparador (véase la Tabla } 2 \text { para definiciones } \\
\text { generales de estas intervenciones) }\end{array}$ & $\begin{array}{l}\text { Resultados en términos de } \\
\text { eficiencia* }\end{array}$ & Referencias \\
\hline $\begin{array}{l}\text { Facilitación de acceso a una página web interactiva } \\
(\mathrm{n}=1)\end{array}$ & $\begin{array}{l}\text { Intervención breve presencial por parte del médico de } \\
\text { cabecera }(n=1)\end{array}$ & Rentable & Hunter R et al., 2017 \\
\hline $\begin{array}{l}\text { Programa de prevención de abuso de alcohol } \\
\text { (AMPP) }(n=3)\end{array}$ & Grupo de control histórico $(\mathrm{n}=1)$ & Rentable y coste-beneficio & Li T et al., 2017 \\
\hline Atención escalonada $(n=2)$ & Intervención mínima grupo de control (n=2) & Dominante o rentable & $\begin{array}{l}\text { Coulton S et al., 2017; } \\
\text { Drummond C et al., } 2009\end{array}$ \\
\hline $\begin{array}{l}\text { SBIRT (cribado, intervención breve y derivación a } \\
\text { tratamiento) en servicio hospitalario de urgencias } \\
\text { (incluidos centros de trauma) }(n=1)\end{array}$ & $\begin{array}{l}\text { SBIRT en entornos sanitarios ambulatorios (centros } \\
\text { sanitarios homologados por el estado o clínicas } \\
\text { hospitalarias de atención ambulatoria) }(n=1)\end{array}$ & Rentable & Barbosa C et al., 2015 \\
\hline $\begin{array}{l}\text { Intervención escolar en línea de varias sesiones basado } \\
\text { en el modelo I-Change }(n=1)\end{array}$ & $\begin{array}{l}\text { Atención habitual (CAU) }(n=1) \text { (lista de espera como } \\
\text { condición de control) }\end{array}$ & $\begin{array}{l}\text { Rentable para subgrupos } \\
\text { poblacionales }\end{array}$ & Drost RM et al., 2016 \\
\hline \multicolumn{4}{|l|}{ Intervenciones combinadas $(n=7)$} \\
\hline $\begin{array}{l}\text { Intervención breve + derivación a servicios de } \\
\text { tratamiento por uso de alcohol }(n=1)\end{array}$ & $\begin{array}{l}\text { Identificación oportunista y grupo de control de } \\
\text { información solo }(n=1)\end{array}$ & Rentable & Barrett B et al., 2006 \\
\hline $\begin{array}{l}\text { Intervención breve + folleto informativo de la salud } \\
(\mathrm{n}=1)\end{array}$ & Folleto informativo de la salud $(n=1)$ & Rentable & Kunz FM et al., 2004 \\
\hline $\begin{array}{l}\text { Intervenciones breves + telemarketing de médicos de } \\
\text { cabecera + apoyo de médicos de cabecera }(n=1)\end{array}$ & Pruebas de alcoholemia aleatorias $(n=1)$ & Rentable & Cobiac L et al., 2009 \\
\hline $\begin{array}{l}\text { Atención habitual mejorada (EUC) + Terapia para } \\
\text { problemas relacionados con el alcohol (CAP) }(n=2)\end{array}$ & EUC solo $(n=2)$ & Dominante o rentable & Nadkarni et al., 2017a, 2017b \\
\hline $\begin{array}{l}\text { Una intervención universal combinada escolar y parental } \\
\text { para la prevención del abuso de alcohol: Steps Towards } \\
\text { Alcohol Misuse Prevention Programme (STAMPP) }(n=1)\end{array}$ & Grupo de control: Currículo escolar habitual (EAN) $(n=1)$ & Rentable & Sumnall H et al., 2017 \\
\hline $\begin{array}{l}\text { Intervención de currículo universal escolar para la } \\
\text { prevención del abuso de alcohol + intervención breve } \\
\text { parental }(n=1)\end{array}$ & Currículo escolar habitual (EAN) (n=1) & Rentable & Agus A et al., 2019 \\
\hline \multicolumn{4}{|c|}{ C: Intervenciones políticas, legislativas y de aplicación $(n=44)$} \\
\hline \multirow{3}{*}{ Control/prohibición de la publicidad $(n=6)$} & Intervención breve $(\mathrm{n}=1)$ & Dominante & Holm AL et al., 2014b \\
\hline & Pruebas de alcoholemia aleatorias $(n=1)$ & Dominante & Cobiac L et al., 2009 \\
\hline & Sin intervención o Situación actual (c) (n= 4) & $\begin{array}{l}\text { Dominante o rentable } \\
(n=3) \text { Dominado }(n=1)\end{array}$ & $\begin{array}{l}\text { Chisholm D et al., } 2004(n=3) \text {; } \\
\text { Lai T et al., } 2007\end{array}$ \\
\hline \multirow{2}{*}{ Pruebas de alcoholemia aleatorias $(n=4)$} & Situación actual $(c)(n=1)$ & Dominado & Lai T et al., 2007 \\
\hline & Sin intervención $(n=3)$ & Dominante o rentable & Chisholm D et al., $2004(n=3)$ \\
\hline $\begin{array}{l}\text { Tasa que mantiene la pérdida de eficiencia actual } \\
\text { de los impuestos }(n=1)\end{array}$ & & Dominante & \\
\hline $\begin{array}{l}\text { Tasa que mantiene los impuestos existentes } \\
\text { Ingresos }(\mathrm{n}=1)\end{array}$ & Sistema de impuestos existente $(n=3)$ & Dominante & Byrnes JM et al., 2010 \\
\hline $\begin{array}{l}\text { Tasa igual a la tasa existente aplicada } \\
\text { sobre las bebidas alcohólicas }(n=1)\end{array}$ & & Dominante & \\
\hline \multirow{4}{*}{ Subida de impuestos $(\mathrm{n}=11)$} & Intervención breve $(\mathrm{n}=1)$ & Dominante & Holm AL et al., 2014b \\
\hline & Pruebas de alcoholemia aleatorias $(n=1)$ & Dominante & Cobiac L et al., 2009 \\
\hline & Sin intervención o Situación actual (c) (n = 8) & $\begin{array}{l}\text { Dominante }(n=1) \\
\text { dominante o rentable }(\mathrm{n} \\
=4) \\
\text { Rentable }(\mathrm{n}=2)\end{array}$ & $\begin{array}{l}\text { Chisholm D et al., } 2004(\mathrm{n}=3) \text {; } \\
\text { Holm AL et al., 2014a; Lai T et } \\
\text { al., 2007; van den Berg M et al., } \\
\text { 2008; Chisholm D et al., } 2018\end{array}$ \\
\hline & $\begin{array}{l}\text { Precio base mínimo unitario aplicado a todo tipo de } \\
\text { bebidas alcohólicas }(n=1)\end{array}$ & Dominante & Robinson E et al., 2020 \\
\hline \multirow{3}{*}{ Control de licencias $(n=6)$} & Intervención breve $(\mathrm{n}=1)$ & Dominante & Holm AL et al., 2014b \\
\hline & Pruebas de alcoholemia aleatorias $(n=1)$ & Rentable & Cobiac L et al., 2009 \\
\hline & Sin intervención o Situación actual (c) (n = 4) & $\begin{array}{l}\text { Dominante }(n=3) \\
\text { Dominado }(n=1)\end{array}$ & $\begin{array}{l}\text { Chisholm D et al., } 2004(n=3) \text {; } \\
\text { Lai T et al., } 2007\end{array}$ \\
\hline \multirow{2}{*}{ Edad legal para beber $(n=2)$} & Intervención breve $(\mathrm{n}=1)$ & Rentable & Holm AL et al., 2014b \\
\hline & Pruebas de alcoholemia aleatorias $(n=1)$ & Dominante & Cobiac L et al., 2009 \\
\hline $\begin{array}{l}\text { Campañas en los medios de comunicación } \\
(\mathrm{n}=3)\end{array}$ & Pruebas de alcoholemia aleatorias $(n=3)$ & Rentable & $\begin{array}{l}\text { Cobiac L et al., 2009; Chisholm } \\
\text { D et al., } 2018\end{array}$ \\
\hline $\begin{array}{l}\text { Promulgación y aplicación de legislación de la } \\
\text { conducción bajo los efectos del alcohol y } \\
\text { los límites de concentración de alcohol en sangre } \\
\text { (a través de controles de alcoholemia) }(n=2)\end{array}$ & Sin intervención o Situación actual $(n=2)$ & Rentable & Chisholm D et al., 2018 \\
\hline $\begin{array}{l}\text { Promulgación y aplicación de legislación de } \\
\text { restricciones a la disponibilidad física de puntos de } \\
\text { venta de alcohol (a través de la reducción de los } \\
\text { horarios de venta) }(n=2)\end{array}$ & Sin intervención o Situación actual $(n=2)$ & Rentable & Chisholm D et al., 2018 \\
\hline
\end{tabular}


Tabla 3. (cont.).

\begin{tabular}{|c|c|c|c|}
\hline Intervención & $\begin{array}{l}\text { Comparador (véase la Tabla } 2 \text { para definiciones } \\
\text { generales de estas intervenciones) }\end{array}$ & $\begin{array}{l}\text { Resultados en términos de } \\
\text { eficiencia* }\end{array}$ & Referencias \\
\hline \multicolumn{4}{|l|}{ Intervenciones combinadas $(n=5)$} \\
\hline $\begin{array}{l}\text { Subida de impuestos + controles de la publicidad } \\
(n=4)\end{array}$ & $\begin{array}{l}\text { Sin intervención o Situación actual (c) } \\
(n=4)\end{array}$ & Rentable & $\begin{array}{l}\text { Chisholm D et al., } 2004(n=3) \text {; } \\
\text { Lai T et al., } 2007\end{array}$ \\
\hline $\begin{array}{l}\text { Subida de impuestos + pruebas de alcoholemia } \\
\text { aleatorias }(n=1)\end{array}$ & $\begin{array}{l}\text { Situación actual (c) } \\
(\mathrm{n}=1)\end{array}$ & Dominado & Lai T et al.,2007 \\
\hline \multicolumn{4}{|l|}{ A y B $(n=22)$} \\
\hline $\begin{array}{l}\text { Entrevista motivacional + informe de retroalimentación } \\
(\mathrm{n}=1)\end{array}$ & $\begin{array}{l}\text { Entrevista motivacional + informe de retroalimentación } \\
(>3 \text { meses })(n=1)\end{array}$ & Rentable & Cowell AJ et al., 2012 \\
\hline $\begin{array}{l}\text { Terapia cognitivo-conductual grupal para mujeres } \\
(\mathrm{G}-\mathrm{FS}-\mathrm{CBT})(\mathrm{n}=1)\end{array}$ & $\begin{array}{l}\text { Terapia cognitivo-conductual individual para mujeres } \\
(\text { I-FS-CBT) }(n=1)\end{array}$ & Rentable & Olmstead TA et al., 2019 \\
\hline $\begin{array}{l}\text { Terapia de estímulo de la motivación + intervenciones } \\
\text { breves + derivación a servicios locales especializados } \\
\text { en el tratamiento por uso de alcohol }(n=1)\end{array}$ & Intervención breve + folleto informativo ( $\mathrm{n}=1)$ & Dominante o rentable & Watson J et al., 2013 \\
\hline $\begin{array}{l}\text { Intervenciones breves + Terapia de facilitación de } 12 \\
\text { pasos + Terapia matrimonial, de pareja o de familia } \\
+ \text { Terapia grupal + Intervenciones farmacológicas (no } \\
\text { especificadas por tipo) + Prevención de recaída + } \\
\text { Citas con médico (cuatro veces la intensidad de las } \\
\text { intervenciones que el grupo de control) }(n=1)\end{array}$ & $\begin{array}{l}\text { Intervenciones breves + Terapia de facilitación de } 12 \\
\text { pasos + Terapia matrimonial, de pareja o de familia } \\
\text { + Terapia grupal + Intervenciones farmacológicas (no } \\
\text { especificadas por tipo) + Prevención de recaída + Citas } \\
\text { con médico }(n=1)\end{array}$ & ne & Weisner C et al., 2000 \\
\hline $\begin{array}{l}\text { Intervenciones breves + Terapia cognitivo-conductual } \\
+ \text { Terapia matrimonial, de pareja o de familia + } \\
\text { Acamprosato }(n=2)\end{array}$ & Sin intervención $(n=2)$ & Rentable & Corry J et al., $2004(n=2)$ \\
\hline $\begin{array}{l}\text { Contacto autoinformado para el problema de salud } \\
\text { mental + Terapia cognitivo-conductual + Intervención } \\
\text { breve+Acamprosato }(n=2)\end{array}$ & Sin intervención $(n=2)$ & Rentable & Corry J et al., $2004(n=2)$ \\
\hline Acamprosato + Intervención breve $(n=1)$ & Intervención breve $(\mathrm{n}=1)$ & Dominante & Palmer Al et al., 2000 \\
\hline $\begin{array}{l}\text { Intervención breve (terapia semanal) + Opioide o } \\
\text { antagonista opiáceo }(\mathrm{n}=1)\end{array}$ & Intervención breve + Placebo $(n=1)$ & ne & Mortimer D y Segal L, 2005 \\
\hline $\begin{array}{l}\text { Cribado + Intervención breve (IB) por parte del médico } \\
\text { de cabecera }(n=11)\end{array}$ & Cribado solo $(n=11)$ & Rentable & Navarro HJ et al., 2011 \\
\hline $\begin{array}{l}\text { Intervención breve para problemas con el } \\
\text { alcohol }(n=1)\end{array}$ & Atención habitual $(n=1)$ & Rentable & Gentilello EL et al., 2019 \\
\hline \multicolumn{4}{|l|}{ B y C $(n=7)$} \\
\hline $\begin{array}{l}\text { Intervención breve + subida de impuestos } \\
(\mathrm{n}=1)\end{array}$ & Situación actual (c) $(\mathrm{n}=1)$ & Dominado & Lai T et al., 2007 \\
\hline $\begin{array}{l}\text { Subida de impuestos + Controles de la publicidad } \\
+ \text { Intervención breve }(n=4)\end{array}$ & Sin intervención o Situación actual (c) $(n=4)$ & $\begin{array}{c}\text { Dominado }(n=1) \\
\text { ne }(n=3)\end{array}$ & $\begin{array}{l}\text { Chisholm D et al., } 2004(n=3) \text {; } \\
\text { Lai T et al., } 2007\end{array}$ \\
\hline $\begin{array}{l}\text { Subida de impuestos + Controles de la publicidad } \\
+ \text { Pruebas de alcoholemia aleatorias + Control de } \\
\text { licencias + Intervención breve }(n=1)\end{array}$ & Situación actual (c) $(\mathrm{n}=1)$ & Rentable & Lai T et al., 2007 \\
\hline $\begin{array}{l}\text { Subida de impuestos + Controles de la publicidad } \\
+ \text { Intervención breve }(\mathrm{n}=1)\end{array}$ & Situación actual (c) $(n=1)$ & Dominado & Lai T et al., 2007 \\
\hline
\end{tabular}

Nota. (a) «Práctica actual» se define en los Países Bajos como la atención habitual. Los autores no especifican en qué consiste esta atención habitual.

(b) «Control» se define como una estrategia en la cual no hay una formación inicial ni apoyo continuo respecto de la implementación del programa.

(c) «Situación actual» se define como «no hacer algo contrafactual», una situación en la cual no hay intervención alguna.

ne: información no especificada.

*Dominante: la intervención o el tratamiento nuevo resulta tener mayor rentabilidad y eficiencia, por lo que se obtiene mayor salud con menos coste. Esto significa que la intervención o el tratamiento nuevo tiene dominancia sobre el comparador. Dominado: la intervención o el tratamiento nuevo resulta tener menor eficiencia y mayor coste; esto significa que el comparador tiene dominancia sobre la intervención o el tratamiento nuevo.

Respecto a los tratamientos para personas con dependencia al alcohol $(\mathrm{n}=63)$, el $29,5 \%$ se centró en intervenciones psicosociales; el $9,8 \%$ en intervenciones farmacológicas; el 1,6\% evaluó otras intervenciones como el tratamiento residencial; y el 59,0 \% restante evaluó una combinación de estos tipos de intervenciones. En cuanto a las intervenciones psicosociales, a pesar del bajo número de estimaciones en este caso $(n=18)$, pareció que cuando cualquiera de estos tipos de intervenciones (i.e., entrevista motivacional; entrenamiento en autocontrol conductual; entrenamiento en habilidades sociales/de afrontamiento; etc.) se comparó con la ausencia de intervención, que entonces la intervención fue dominante, lo que significa que la intervención fue más eficaz y menos costosa que el comparador. De manera similar, cabe destacar el estudio realizado por Slattery et al. (2002) quienes encontraron que diferentes intervenciones psicosociales para las personas con dependencia al alcohol fueron dominantes, lo que resultó en ahorros entre $923 £$ y $1643 £$ por paciente adicional en abstinencia en comparación con el tratamiento estándar. Sin embargo, ninguna intervención específica mostró una clara tendencia en términos de eficiencia en comparación 
con otra. El bajo número de evaluaciones económicas de intervenciones farmacológicas $(\mathrm{n}=7)$ y la heterogeneidad de la evidencia hallada resultó en la imposibilidad de establecer conclusiones en términos de eficiencia para este tipo de programas. Aunque el número de comparaciones de intervenciones exclusivamente farmacológicas fue bajo, hubo algunas evaluaciones económicas de intervenciones combinadas que incluyen fármacos. Por tanto, estos estudios se han incluido en las intervenciones farmacológicas. Así, se han añadido 34 comparaciones basadas principalmente en el tratamiento con acamprosato, un modulador del receptor de N-metil-D-aspartato (NMDA) y opioides o antagonistas opiáceos como el nalmefeno. A pesar del mayor número de comparaciones existentes al incluir las intervenciones combinadas que incluían fármacos, no se pudieron encontrar resultados concluyentes relacionados con el acamprosato. El uso de acamprosato exclusivamente para el tratamiento de la dependencia al alcohol ha mostrado ser dominante o rentable en las tres comparaciones con uso de placebo. Cuando se usó en combinación con intervención psicológica y tratamiento médico, tuvo dominancia el tratamiento médico y el placebo, así como la combinación de tratamiento médico, opioides o antagonistas opiáceos y acamprosato. Por tanto, cuando se usó en combinación con un opioide o antagonista opiáceo, fue rentable o dominante. Asimismo, cuando la combinación de tratamiento médico con acamprosato se comparó con tratamiento médico y placebo, así como con tratamiento médico y opioides o antagonistas opiáceos, estas últimas combinaciones eran dominantes. Sin embargo, en relación con el uso de nalmefeno combinado con terapia psicológica, demostró su rentabilidad o dominancia en comparación con terapia psicológica solo o placebo en las 12 comparaciones halladas en esta revisión (Brodtkorb, Bell, Irving y Laramée, 2016; Laramée, Bell, Irving, y Brodtkorb, 2016; Laramée et al., 2014; y Millier et al., 2017). Lo mismo se aplica a la evaluación económica de las intervenciones combinadas en este caso (ver Tabla 3).

Respecto de los tratamientos para personas con factores de alto riesgo de tener dependencia al alcohol, la intervención breve ha sido la más evaluada (47,8 \%). Al comparar esta intervención con la ausencia de intervención, a pesar de la escasa evidencia, pareció que la intervención breve (en este caso centrada principalmente en asesoramiento breve) podría ser una estrategia dominante o rentable. Asimismo, cuando este tipo de intervención se aplicó en entornos distintos, p. ej., según el estudio realizado por Barbosa, Cowell, Bray y Aldridge (2015), la intervención combinada conocida como SBIRT que incluye intervención breve resulta en ahorros de costes y mejoras en la salud tanto en servicios de emergencia como ambulatorios, siendo más eficiente, lo que significa que brinda mayor efectividad a menor coste en servicios de urgencias que en entornos ambulatorios. No se pudieron extraer otras con- clusiones de otras intervenciones o intervenciones combinadas (p. ej., intervención breve + derivación a servicios de tratamiento por uso de alcohol) en términos de eficiencia debido a la inconsistencia de la evidencia encontrada (ver Tabla 3).

En cuanto a las intervenciones políticas, legislativas y de aplicación, se reconoce que podría haber una tendencia a intervenciones del tipo de control de la publicidad, pruebas aleatorias de alcoholemia, subidas de impuestos y control de licencias, dada su dominancia o rentabilidad en comparación con la ausencia de intervención. Por ejemplo, en el estudio realizado por Chisholm et al. (2018), un aumento del $50 \%$ en las tasas de los impuestos sobre el consumo resultó en un bajo coste de implementación (menos de I \$ 0.10 per cápita), un nivel de impacto en la salud traducido en más de 500 años de vida saludable ganados por millón de habitantes y un nivel muy favorable en términos de la ratio de coste-efectividad, que es menos de I \$ 100 por año de vida saludable ganado, siendo esta la estrategia de intervención más rentable entre las evaluadas (ver Tabla 3).

\section{Discusión}

Algunas revisiones de la literatura sobre las medidas para reducir el abuso de alcohol se han publicado anteriormente (Angus et al., 2014; Barbosa et al., 2010; Chisholm et al., 2006; Hill et al., 2017; Hoang et al., 2016; Kaner et al., 2017; Kelly et al., 2020; Kruse et al., 2020; Ludbrook, 2004; Ludbrook et al., 2002; Meads et al., 2007; Mujoomdar y Spry, 2009; Ndegwa y Cunningham, 2009; Poldrugo et al., 2005; Rehm y Barbosa, 2018; Slattery et al., 2002; White et al., 2018; Yadav y Kobayashi, 2015); sin embargo, nadie ha abordado la cuestión de identificar qué programa o intervención fue más eficiente en términos de tratamientos para personas con TUA, aunque Ludbrook (2004) analizó todos los posibles enfoques relacionados con el alcohol, desde el tratamiento hasta la prevención, y las intervenciones desde el nivel individual hasta los enfoques poblacionales. Además, Rehm y Barbosa (2018) concluyeron que la investigación económica hasta la fecha es relativamente escasa y no siempre rigurosa. Por tanto, esta información es necesaria para informar el debate de política sanitaria para determinar el nivel necesario de recursos para abordar los problemas relacionados con el alcohol. La combinación de tratamiento/intervención temprana .e intervenciones de política sanitaria ofrece a los lectores una descripción general de la gama de opciones para impactar el TUA y su potencial en términos de coste-efectividad. Este manuscrito define un punto de partida para los tomadores de decisiones al permitirles priorizar los tipos de intervenciones que tienen mayor potencial de ser eficientes. Este análisis también señala áreas (tratamiento asistido por medicamentos) donde evaluaciones económicas adicionales son necesarias. 
Algunas conclusiones pueden extraerse a pesar de que no se ha encontrado mucha evidencia en términos de eficiencia en esta revisión sistemática de evaluaciones económicas de intervenciones para personas con trastornos por uso de alcohol o personas en riesgo de problemas relacionados con el alcohol. Desafortunadamente, la amplia variedad de medidas de resultado y costes no permite a los tomadores de decisiones escoger la intervención más eficiente. Es imposible determinar si las diferencias en el coste por unidad (p. ej., calidad de vida) ganado se deben realmente a diferencias en la eficiencia de las intervenciones más que a diferencias en los métodos utilizados para las comparaciones; por tanto, la mayoría de las conclusiones a las que se puede llegar se limitan a las intervenciones incluidas en cada estudio por separado. Sin embargo, esta información podría ser útil en la práctica clínica para destacar la importancia de la necesidad de evaluar todas las intervenciones en términos de eficiencia. También muestra cuales intervenciones se han evaluado con mayor frecuencia y cuáles son las variables más importantes a tener en cuenta para realizar evaluaciones económicas de los programas relacionados con el alcohol. En relación a los estudios que evalúan la eficacia de las intervenciones farmacológicas relacionadas con la dependencia al alcohol, se podrían establecer conclusiones sobre el uso de nalmefeno pero no en relación al acamprosato debido a los resultados controvertidos y diferentes obtenidos en los estudios encontrados. Recientemente, Avanceña, Miller, Uttal, Hutton y Mellinger (2020) han hecho una evaluación económica basada en la literatura existente y encontraron que el uso de acamprosato y naltrexona, así como el uso de baclofeno, gabapentina y topiramato, en comparación con la ausencia de intervención, son intervenciones que ahorran costes en pacientes con cirrosis relacionada con el alcohol.

En cuanto a las conclusiones referentes a la intervención breve, esta recomendación ya se estableció para Escocia (Ludbrook, 2004) hace tiempo. Este resultado se mantuvo en nuestra revisión. A pesar de ello, algunos autores (Falcón et al., 2018) describen barreras a la implementación de cribado e intervención breve por uso de alcohol en algunos entornos, como los servicios hospitalarios de urgencias. Debemos señalar que no muchos estudios relacionados con el consumo de sustancias para dejar el uso de alcohol o para ayudar a reducir la ingesta de alcohol se han evaluado desde el punto de vista de la eficiencia. Por tanto, existe la necesidad de que la industria farmacéutica, que produce medicamentos que reducen la ingesta de alcohol, invierta en medir y evaluar la eficiencia de sus productos para reducir la ingesta de alcohol y disminuir la recaída al consumo excesivo de alcohol. Además, esta revisión sistemática no ha identificado estudio alguno dirigido a mejorar el funcionamiento cognitivo en pacientes con deterioro cognitivo asociado al uso de alcohol que están en tratamiento para la dependencia al alcohol. Sin embar- go, autores como Frías-Torres et al. (2018) sugieren cómo la terapia de rehabilitación cognitiva podría mejorar esta condición.

Hay otras revisiones de evaluaciones económicas centradas en las intervenciones farmacológicas, como el uso de naltrexona (Mujoomdar y Spry, 2009), los instrumentos políticos (Chisholm et al., 2006), el cribado y las intervenciones breves (Angus et al., 2014) y la evaluación de métodos para evaluaciones económicas de tratamientos para TUA (Barbosa et al., 2010). Sin embargo, en realidad no evaluaron la eficiencia de esos programas. Esta revisión mantiene las conclusiones de Brown et al. (2016) quienes mostraron que había una escasez de evaluaciones de la efectividad de las intervenciones farmacológicas para el tratamiento del uso de alcohol. El presente estudio incluyó todas las referencias proporcionadas por estas revisiones, con la excepción de algunas que no eran evaluaciones económicas completas y, por tanto, no proporcionaron una RCEI (Alwyn, John, Hodgson y Phillips, 2004; Babor et al., 2006; Bischof et al., 2008; Humphreys y Moos, 1996; Lock et al., 2006; Long, Williams y Hollin, 1998; Nalpas et al., 2003; Pettinati et al., 1999; Shakeshaft, Bowman, Burrows, Doran y Sanson-Fisher, 2002; Sobell et al., 2002). Además, fomenta la idea de pensar, en todas estas intervenciones, en términos de tratar a las personas con dependencia al alcohol; tratar a las personas en riesgo de problemas relacionados con el alcohol, e intervenciones políticas, legislativas y de aplicación. Por tanto, si los decisores se planteaban implementar un programa potencial en un país específico, las intervenciones recomendables según criterios de eficiencia serían cualquier intervención psicosocial, intervenciones breves para personas en riesgo de problemas relacionados con el alcohol y controles de la publicidad, subidas de impuestos, control de licencias, edad legal para beber y campañas en los medios de comunicación. Así, la información generada por esta revisión sistemática sería útil para la toma de decisiones respecto de las intervenciones en las cuales invertir recursos de salud pública para abordar la rehabilitación de los trastornos relacionados con el alcohol.

En coherencia con las intervenciones políticas, legislativas y de aplicación, dado los hallazgos favorables en términos de la ratio de coste-efectividad incremental, parece recomendable que los países impulsen este tipo de intervenciones para mejorar la eficiencia de este problema de salud pública. Es sorprendente la falta de evaluaciones económicas de intervención preventiva basada en un «precio mínimo unitario» o "precio unitario mínimo» (PUM). Por un lado, Lonsdale, Hardcastle y Hagger (2012) concluyeron, a través de grupos focales, que los participantes plantearon una serie de objeciones relacionadas con el escepticismo sobre si el PUM es un medio eficaz para reducir el consumo de alcohol, la percepción de que la política «castiga» al bebedor moderado y la preocupación de que esta medida pueda agravar los problemas sociales existen- 
tes. Además, los participantes del estudio expresaron que esta medida podría funcionar si fuese parte de una campaña más amplia que incluyese otras actividades educativas. Asimismo, Purshouse, Meier, Brennan, Taylor y Rafia (2010) concluyeron que el modelo de política de alcohol de Sheffield (Holmes et al., 2014) predice que el establecimiento de un PUM reduciría los daños relacionados con el alcohol, en mayor medida que las subidas generales de impuestos, y así casi doblar la prevención de muertes.

Además, hubo interés por comparar la eficiencia de diferentes intervenciones según el nivel de dependencia al alcohol (i.e., la eficiencia de las intervenciones dirigidas a las personas con dependencia al alcohol entre moderada y grave, en comparación con las intervenciones dirigidas a problemas de menor gravedad en relación al alcohol). Sin embargo, las definiciones utilizadas en los estudios para clasificar la dependencia al alcohol han sido diferentes (i.e., personas con una puntuación en el AUDIT > 8; personas que beben $>200 \mathrm{~g} /$ día). Por tanto, sin una definición homogénea no es posible estudiar el impacto sobre los resultados según los diferentes grados de dependencia al alcohol. En relación a la tendencia observada en el uso y la eficiencia de las intervenciones, tales como control de la publicidad, pruebas de alcoholemia aleatorias, subidas de impuestos y control de licencias, parece ser recomendable que los países promuevan este tipo de intervenciones para mejorar la eficiencia en este problema de salud pública. Son necesarios más estudios para caracterizar los umbrales de rentabilidad en el campo del uso de sustancias. Para hacerlo, es necesario proporcionar más evidencia en términos de coste-efectividad de todas estas intervenciones diferentes para abordar la dependencia al alcohol. Sin embargo, es necesario evaluar cuánto está dispuesta la sociedad a pagar por este tipo de intervenciones y la mejora de los resultados de salud generados. Por tanto, esta cuestión podría explorarse mediante estudios sobre la disposición a pagar o experimentos de elección discreta.

Una de las limitaciones de esta revisión es el número limitado de estudios encontrados de los cuales extraer conclusiones. Idealmente, estas conclusiones deberían haberse extraído de acuerdo con el país del estudio para asegurar la aplicabilidad de los resultados a cada contexto específico. Por tanto, esta revisión todavía sugiere que son necesarios más estudios para evaluar la eficiencia de las intervenciones y los programas para reducir el abuso de alcohol a nivel global. Barbosa et al. (2010) señalaron hace algunos años que este tipo de literatura todavía escaseaba y se requería más investigación para llenar los vacíos. Continúa siendo necesario utilizar una metodología común en las futuras evaluaciones económicas del tratamiento del alcohol para producir estimaciones de coste-efectividad más estables y para informar las decisiones para la asignación de recursos para un tratamiento eficiente del uso de alcohol. Otro tema planteado por esta revisión sistemática de la literatura es que muy pocos estudios consideraron los costes directos para el paciente y las pérdidas de productividad, entre otros costes, refiriéndose principalmente a efectos externos como la justicia penal, los servicios de bomberos o los accidentes mortales en estudios para el tratamiento de los trastornos por uso de alcohol o personas en riesgo de problemas relacionados con el alcohol.

No solo es necesario realizar más investigaciones sobre la eficiencia, sino también sobre la eficacia de los diferentes programas o intervenciones. Según Yadav y Kobayashi (2015), a pesar de la década adicional de evidencia, los estudios disponibles tenían enfoques heterogéneos, por lo que no se pudieron generar conclusiones sobre la efectividad de las campañas en los medios de comunicación. Se necesitan más estudios en términos de efectividad y rentabilidad para evaluar los programas relacionados con la ingesta de alcohol. Además, también era necesario informar mejor la metodología de costes de los diferentes estudios (Bray, Zarkin, Hinde y Mills, 2012). Los costes relacionados con la evaluación de programas, como el cribado de alcohol y la intervención breve en entornos médicos, pueden presentar grandes diferencias porque no se estableció de forma común la metodología de costes.

De hecho, es necesario fomentar una cultura de evaluación entre los responsables de la prestación de servicios y diseñar algunas pautas para promover esta cultura de evaluación en los programas de salud pública (Ludbrook, 2004). Debe prestarse especial atención a la evaluación de la eficiencia de los programas relacionados con el alcohol.

\section{Reconocimientos}

Los autores están muy agradecidos a la Dra. Kathryn McCollister del Departamento de Ciencias de la Salud Pública de la Facultad de Medicina Miller de la Universidad de Miami por sus comentarios útiles en relación a este manuscrito.

\section{Conflicto de interés}

Este proyecto fue parcialmente financiado por el Ministerio de Economía y Competitividad (MINECO) de España bajo el Programa Estatal de Investigación, Desarrollo e Innovación Orientada a los Retos de la Sociedad, Plan Estatal de Investigación Científica Técnica y de Innovación 2013-2016. Subvención ECO2013-48217-C2-1 (http://invesfeps.ulpgc.es/en). También ha sido parcialmente financiado por la Agència de gestió d'Ajuts Universitaris i de Recerca (AGAUR) en el marco del programa «Suports a Grups de Recerca (SGR 2017-2019)» del grupo: «Grup de Recerca en Avaluació de Polítiques Públiques», expediente 2017 SGR 263. El financiador no influyó en la realización de este estudio ni en la redacción de este manuscrito. 


\section{Referencias}

Alwyn, T., John, B., Hodgson, R. J. y Phillips, C. J. (2004). The addition of a psychological intervention to a home detoxification programme. Alcohol and Alcoholism, 39, 536-541. doi:10.1093/alcalc/agh092.

Angus, C., Latimer, N., Preston, L., Li, J. y Purshouse, R. (2014). What are the implications for policy makers? A systematic review of the cost-effectiveness of screening and brief interventions for alcohol misuse in primary care. Frontiers in Psychiatry, 5, 114. doi:10.3389/ fpsyt.2014.00114.

Avanceña, A., Miller, N., Uttal, S. E., Hutton, D. W. y Mellinger, J. L. (2020). Cost-effectiveness of alcohol use treatments in patients with alcohol-related cirrhosis. Journal of hepatology. Advance publication online. doi:10.1016/j. jhep.2020.12.004.

Babor, T. F., Higgins-Biddle, J. C., Dauder, D., Burleson, J. a., Zarkin, G. a. y Bray, J. (2006). Brief interventions for at-risk drinking: Patient outcomes and cost-effectiveness in managed care organizations. Alcohol and Alcoholism, 41, 624-631. doi: 10.1093/alcalc/agl078.

Barbosa, C., Cowell, A., Bray, J., y Aldridge, A. (2015). The cost-effectiveness of alcohol Screening, Brief Intervention, and Referral to Treatment (SBIRT) in emergency and outpatient medical settings. Journal of Substance Abuse Treatment, 53, 1-8. doi:10.1016/j.jsat.2015.01.003.

Barbosa, C., Cowell, A., Dowd, W., Landwehr, J., Aldridge, A., y Bray, J. (2017). The cost-effectiveness of brief intervention versus brief treatment of Screening, Brief Intervention and Referral to Treatment (SBIRT) in the United States. Addiction, 112 (Supl. 2), 73-81. doi:10.1111/ add.13658.

Barbosa, C., Godfrey, C. y Parrott, S. (2010). Methodological assessment of economic evaluations of alcohol treatment: What is missing? Alcohol and Alcoholism, 45, 53-63. doi:10.1093/alcalc/agp067.

Bischof, G., Grothues, J. M., Reinhardt, S., Meyer, C., John, U. y Rumpf, H. J. (2008). Evaluation of a telephone-based stepped care intervention for alcohol-related disorders: A randomized controlled trial. Drug and Alcohol Dependence, 93, 244-251. doi:10.1016/j.drugalcdep.2007.10.003.

Bray, J. W., Zarkin, G. A., Hinde, J. M. y Mills, M. J. (2012). Costs of alcohol screening and brief intervention in medical settings: a review of the literature. Journal of Studies on Alcohol and Drugs, 73, 911-919. doi:10.15288/ jsad.2012.73.911.

Brodtkorb, T-H., Bell, M., Irving, A.H. y Laramée, P. (2016). The cost effectiveness of nalmefene for reduction of alcohol consumption in alcohol-dependent patients with high or very high drinking-risk levels from a UK societal perspective. CNS Drugs, 30, 163-177. doi:10.1007/ s40263-016-0310-2.
Brown, T. J., Todd, A., O’Malley, C. L., Moore, H. J., Husband, A. K., Bambra, C.,... Summerbell, C. D. (2016). Community pharmacy interventions for public health priorities: a systematic review of community pharmacy-delivered smoking, alcohol and weight management interventions. BMJ Open, 6, e009828. doi:10.1136/bmjopen-2015-009828.

Burke, T. R. (1988). The economic impact of alcohol abuse and alcoholism. Public Health Reports, 103, 564-568.

Chisholm, D., Doran, C., Shibuya, K. y Rehm, J. (2006). Comparative cost-effectiveness of policy instruments for reducing the global burden of alcohol, tobacco and illicit drug use. Drug and Alcohol Review, 25, 553-565. doi:10.1080/09595230600944487.

Chisholm, D., Moro, D., Bertram, M., Pretorius, C., Gmel, G., Shield, K. y Rehm, J. (2018). Are the "best buys" for alcohol control still valid? An update on the comparative cost-effectiveness of alcohol control strategies at the global level. Journal of Studies on Alcohol and Drugs, 79, 514-522.

Chisholm, D., Rehm, J., Van Ommeren, M. y Monteiro, M. (2004). Reducing the global burden of hazardous alcohol use: a comparative cost-effectiveness analysis. Journal of Studies on Alcohol, 65, 782-793. doi: 10.15288/ jsa.2004.65.782.

Cobiac, L., Vos, T., Doran, C. y Wallace, A. (2009). Cost-effectiveness of interventions to prevent alcohol-related disease and injury in Australia. Addiction, 104, 1646-1655. doi:10.1111/j.1360-0443.2009.02708.x.

Donaldson, C., Mugford, M. y Vale, L. (Eds.). (2002). Evidence-based health economics: from effectiveness to efficiency in systematic review. London: BMJ Publishing Group.

Drummond, M., Sculpher, M., Claxton, K., Stoddard, G. L. y Torrance, G. W. (2015). Methods for the economic evaluation of health care programmes (4th ed.). United Kingdom: Oxford University Press.

Drummond, M., Weatherly, H., Claxton, K., Cookson, R., Ferguson, B., Godfrey, C. y Rice, N. (2007). Assessing the challenges of applying standard methods of economic evaluation to public health interventions (2nd ed.). York: Public Health Research Consortium.

Dunlap, L. J., O’Farrell, T. J., Schumm, J. A., Orme, S. S., Murphy, M. y Murchowski, P. M. (2020). Group versus standard behavioral couples' therapy for alcohol use disorder patients: Cost-effectiveness. Journal of Studies on Alcohol and Drugs, 81, 152-163. doi:10.15288/ jsad.2020.81.152.

Dunlap, L. J., Zarkin, G. A., Bray, J. W., Mills, M., Kivlahan, D. R., McKay, J. R.,... Tonigan, J. S. (2010). Revisiting the cost-effectiveness of the COMBINE study for alcohol dependent patients: The patient perspective. Medical Care, 48, 306-313. doi:10.1097/mlr.0b013e3181ca3d40.

Falcón, M., Navarro-Zaragoza, J., García-Rodríguez, R. M., Nova-López, D., González-Navarro, M. D., Mercadal, 
M.,... Luna Ruiz-Cabello, A. (2018). Perceived barriers to implementing screening and brief intervention for alcohol consumption by adolescents in hospital emergency department in Spain. Adicciones, 30, 189-196. doi:10.20882/adicciones.896.

Frías-Torres, C., Moreno-España, J., Ortega, L., Barrio, P., Gual, A. y Teixidor López, L. (2018). Remediation therapy in patients with alcohol use disorders and neurocognitive disorders: A Pilot Study. Adicciones, 30, 93-100. doi:10.20882/adicciones.757.

Goeree, R. y Diaby, V. (2013). Introduction to health economics and decision-making: Is economics relevant for the frontline clinician? Best Practice and Research: Clinical Gastroenterology, 27, 831-844. doi: 10.1016/j. bpg.2013.08.016.

Gold, M. R., Siegel, J. E., Russell, L. B. y Weinstein, M. C. (1996). Cost-effectiveness in health and medicine. New York: Oxford University Press.

Hill, S. R., Vale, L., Hunter, D., Henderson, E. y Oluboyede, Y. (2017). Economic evaluations of alcohol prevention interventions: Is the evidence sufficient? A review of methodological challenges. Health Policy, 121, 1249-1262. doi:10.1016/j.healthpol.2017.10.003.

Hoang, V. P., Shanahan, M., Shukla, N., Perez, P., Farrell, M. y Ritter, A. (2016). A systematic review of modelling approaches in economic evaluations of health interventions for drug and alcohol problems. BMC Health Services Research, 16, 127. doi: 10.1186/s12913-016-1368-8.

Holmes, J., Meng, Y., Meier, P. S., Brennan, A., Angus, C., Campbell-Burton, A., ... Purshouse, R.C. (2014). Effects of minimum unit pricing for alcohol on different income and socioeconomic groups: a modelling study. Lancet, 383, 1655-64. doi:10.1016/S0140-6736(13)62417-4.

Humphreys, K. y Moos, R. (1996). Reduced substance-abuse-related health care costs among voluntary participants in Alcoholics Anonymous. Psychiatric Services, 47, 709-713. doi:10.1176/ps.47.7.709.

Kaner, E. F., Beyer, F. R., Garnett, C., Crane, D., Brown, J., Muirhead, C.,... Michie, S. (2017). Personalised digital interventions for reducing hazardous and harmful alcohol consumption in community-dwelling populations. Cochrane Database of Systematic Reviews, 9, CD011479. doi:10.1002/14651858.CD011479.pub2.

Kelly, J. F., Abry, A., Ferri, M. y Humphreys, K. (2020). Alcoholics anonymous and 12-step facilitation treatments for alcohol use disorder: A distillation of a 2020 Cochrane review for clinicians and policy makers. Alcohol and Alcoholism, 55, 641-651. doi:10.1093/alcalc/agaa050.

Kernick, D. P. (2003). Introduction to health economics for the medical practitioner. Postgraduate Medical Journal, 79, 147-150. doi:10.1136/pmj.79.929.147.

Kruse, C. S., Lee, K., Watson, J. B., Lobo, L. G., Stoppelmoor, A. G. y Oyibo, S. E. (2020). Measures of effectiveness, efficiency, and quality of telemedicine in the mana- gement of alcohol abuse, addiction, and rehabilitation: Systematic review. Journal of Medical Internet Research, 22, e13252. doi: 10.2196/13252.

Laramée, P., Bell, M., Irving, A., y Brodtkorb, T. H. (2016). The Cost-effectiveness of the integration of nalmefene within the UK healthcare system treatment pathway for alcohol dependence. Alcohol and Alcoholism, 51, 283-290. doi:10.1093/alcalc/agv140.

Laramée, P., Brodtkorb, T.-H., Rahhali, N., Knight, C., Barbosa, C., Francois,... Rehm, J. (2014). The cost-effectiveness and public health benefit of nalmefene added to psychosocial support for the reduction of alcohol consumption in alcohol-dependent patients with high/very high drinking risk levels: A Markov model. BMJ Open, 4, e005376. doi:10.1136/bmjopen-2014-005376.

Lock, C. A., Kaner, E., Heather, N., Doughty, J., Crawshaw, A., McNamee, P.,... Pearson, P. (2006). Effectiveness of nurse-led brief alcohol intervention: A cluster randomized controlled trial. Journal of Advanced Nursing, 54, 426-439. doi:10.1111/j.1365-2648.2006.03836.x.

Long, C., Williams, M. y Hollin, C. (1998). Treating alcohol problems: A study of programme effectiveness and cost effectiveness according to length and delivery of treatment. Addiction, 93, 561-571. doi:10.1046/j.13600443.1998.93456111.x

Lonsdale, A. J., Hardcastle, S. J. y Hagger, M. S. (2012). A minimum price per unit of alcohol: a focus group study to investigate public opinion concerning UK government proposals to introduce new price controls to curb alcohol consumption. BMC Public Health, 12, 1023. doi:10.1186/1471-2458-12-1023.

Ludbrook, A. (2004). Effective and cost-effective measures to reduce alcohol misuse in Scotland: an update. Edinburgh: Scottish Executive Health Department.

Ludbrook, A., Godfrey, C., Wyness, L., Parrott, S., Haw, S., Napper, M. y van Teijlingen, E. (2002). Effective and cost-effective measures to reduce alcohol misuse in Scotland: a literature review. Edinburgh: Scottish Executive Health Department.

Meads, C., Ting, S., Dretzke, J. y Bayliss, S. (2007). A systematic review of the clinical and cost-effectiveness of psychological therapy involving family and friends in alcohol misuse or dependence. Report Number 65. Birmingham, Department of Public Health and Epidemiology, West Midlands Health Technology Assessment Collaboration ...

Millier, A., Laramée, P., Rahhali, N., Aballéa, S., Daeppen, J. B., Rehm, J., y Toumi, M. (2017). Cost-effectiveness of nalmefene added to psychosocial support for the reduction of alcohol consumption in alcohol-dependent patients with high/very high drinking risk levels: A microsimulation model. Journal of Studies on Alcohol and Drugs, 78, 867-876. doi:10.15288/jsad.2017.78.867.

Moher, D., Shamseer, L., Clarke, M., Ghersi, D., Liberati, A., Petticrew, M.,... the PRISMA-P Group (2015). Pre- 
ferred Reporting Items for Systematic Review and Meta-Analysis Protocols (PRISMA-P) 2015 statement. Systematic Reviews, 4, 1. doi:10.1186/2046-4053-4-1.

Mujoomdar, M. y Spry, C. (2009). Naltrexone for the treatment of alcohol dependence: a review of the clinical and cost-effectiveness. Ottawa: Canadian Agency for Drugs and Technologies in Health (CADTH).. Recuperado de https:// www.cadth.ca/sites/default/files/pdf/L0076_Naltrexone_for_alcohol_dependence.pdf.

Nalpas, B., Combescure, C., Pierre, B., Ledent, T., Gillet, C., Playoust, D.,... Daurès, J. P . (2003). Financial costs of alcoholism treatment programs: a longitudinal and comparative evaluation among four specialized centers. Alcoholism, Clinical and Experimental Research, 27, 51-56. doi:10.1097/01.ALC.0000047301.72437.10.

Ndegwa, S. y Cunningham, J. (2009). Naltrexone in combination with acamprosate for the treatment of alcohol dependence: a review of the clinical and cost-effectiveness. Ottawa: Canadian Agency for Drugs and Technologies in Health (CADTH). Recuperado de https://www.cadth.ca/media/ pdf/L0123_Naltrexone_and_Acamprosate_final.pdf.

Pettinati, H. M., Meyers, K., Evans, B. D., Ruetsch, C. R., Kaplan, F. N.,Jensen, J. M.y Hadley, T. R. (1999). Inpatient alcohol treatment in a private healthcare setting: Which patients benefit and at what cost? American Journal on Addictions, 8, 220-233. doi:10.1080/105504999305839.

Poldrugo, F., Haeger, D. A., Comte, S., Walburg, J. y Palmer, A. J. (2005). A critical review of pharmacoeconomic studies of acamprosate. Alcohol and Alcoholism, 40, 422-430. doi:10.1093/alcalc/agh171.

Purshouse, R. C., Meier, P. S., Brennan, A., Taylor, K. B. y Rafia, R. (2010). Estimated effect of alcohol pricing policies on health and health economic outcomes in England: An epidemiological model. Lancet, 375, 135564. doi:10.1016/S0140-6736(10)60058-X.

Rehm, J. y Barbosa, C. (2018). The cost-effectiveness of therapies to treat alcohol use disorders. Expert review of Pharmacoeconomics E Outcomes Research, 18, 43-49. doi:1 0.1080/14737167.2018.1392241.

Rehm, J., Mathers, C., Popova, S., Thavorncharoensap, M., Teerawattananon, Y. y Patra, J. (2009). Global burden of disease and injury and economic cost attributable to alcohol use and alcohol-use disorders. Lancet, 373, 22232233. doi:10.1016/S0140-6736(09)60746-7.

Shakeshaft, A. P., Bowman, J. A., Burrows, S., Doran, C. M. y Sanson-Fisher, R. W. (2002). Community-based alcohol counselling: A randomized clinical trial. Addiction, 97, 1449-1463. doi:10.1046/j.1360-0443.2002.00199.x.

Slattery, J., Chick, J., Cochrane, M., Craig, J., Godfrey, C., Macpherson, K. y Parrot, S. (2002). Health Technology Assessment of Prevention of Relapse in Alcohol Dependence. Consultation Assessment Report. Scotland: Health Technology Board for Scotland. Recuperado de https:/ /www.drugsan-
dalcohol.ie/19424/1/Health_Technology_Assessment_ Prevention_of_relapse_in_alcohol_dependence.pdf.

Sloan, F. A. y Hsieh, C.-R. (2012). Health Economics. United States of America: The Massachusetts Institute of Technology (MIT) Press.

Smyth, A., Teo, K. K., Rangarajan, S., O’Donnell, M., Zhang, X., Rana, P.,... PURE Investigators (2015). Alcohol consumption and cardiovascular disease, cancer, injury, admission to hospital, and mortality: a prospective cohort study. Lancet, 6736, 1-10.. doi:10.1016/S01406736(15)00235-4.

Sobell, L. C., Sobell, M. B., Leo, G. I., Agrawal, S., Johnson-Young, L. y Cunningh am, J. A. (2002). Promoting self-change with alcohol abusers: a community-level mail intervention based on natural recovery studies. $A l$ coholism: Clinical and Experimental Research, 26, 936-948.

The Joanna Briggs Institute (JBI) (2014). Joanna Briggs Institute for Evidence Based Practice Reviewers' manual 2014. The Systematic Review of Economic Evaluation Evidence. Australia, The Joanna Briggs Institute, The University of Adelaide. Recuperado de https://nursing.lsuhsc.edu/ JBI/docs/ReviewersManuals/Economic.pdf.

White, P., Skirrow, H., George, A. y Memon, A. (2018). A systematic review of economic evaluations of local authority commissioned preventative public health interventions in overweight and obesity, physical inactivity, alcohol and illicit drugs use and smoking cessation in the United Kingdom. Journal of Public Health, 40, e521-e530. doi:10.1093/pubmed/fdy026.

Williams, I., McIver, S., Moore, D. y Bryan, S. (2008). The use of economic evaluations in NHS decision-making: A review and empirical investigation. Health Technology Assessment, 12, 1-175. doi:10.3310/hta12070.

Organización Mundial de la Salud. (1992). The ICD-10 Classification of Mental and Behavioural Disorders: Clinical descriptions and diagnostic guidelines. Geneva, Organización Mundial de la Salud.

Organización Mundial de la Salud (2018). Global status report on alcohol and health 2018. Geneva, Organización Mundial de la Salud.

Yadav, R.-P. y Kobayashi, M. (2015). A systematic review : Effectiveness of mass media campaigns for reducing drink-driving and alcohol-related crashes. BMC Public Health, 15, 857. doi:10.1016/j.amepre.2004.03.002. 


\section{Apéndices}

\section{Apéndice 1. Estudios incluidos en la revisión sistemática $(n=65)$}

Agus, A., McKay, M., Cole, J., Doherty, P., Foxcroft, D., Harvey, S.,... Sumnall, H. (2019). Cost-effectiveness of a combined classroom curriculum and parental intervention: Economic evaluation of data from the Steps Towards Alcohol Misuse Prevention Programme cluster randomised controlled trial. BMJ Open, 9, e027951. doi:10.1136/bmjopen-2018-027951.

Angus, C., Scafato, E., Ghirini, S., Torbica, A., Ferre, F., Struzzo, P.,... Brennan, A. (2014). Cost-effectiveness of a programme of screening and brief interventions for alcohol in primary care in Italy. BMC Family Practice, 15, 26. doi:10.1186/1471-2296-15-26.

Barbosa, C., Cowell, A., Bray, J. y Aldridge, A. (2015). The Cost-effectiveness of Alcohol Screening, Brief Intervention, and Referral to Treatment (SBIRT) in emergency and outpatient medical settings. Journal of Substance Abuse Treatment, 53, 1-8. doi: 10.1016/j.jsat.2015.01.003.

Barbosa, C., Cowell, A., Dowd, W., Landwehr, J., Aldridge, A. y Bray, J. (2017). The cost-effectiveness of brief intervention versus brief treatment of Screening, Brief Intervention and Referral to Treatment (SBIRT) in the United States. Addiction, 112 (Supl. 2), 73-81. doi:10.1111/ add. 13658 .

Barbosa, C., Taylor, B., Godfrey, C., Rehm, J., Parrott, S. y Drummond, C. (2010). Modelling lifetime QALYs and health care costs from different drinking patterns over time: a Markov model. International Journal of Methods in Psychiatric Research, 19, 97-109. doi:10.1002/mpr.306.

Barrett, B., Byford, S., Crawford, M. J., Patton, R., Drummond, C., Henry, J. A. y Touquet, R. (2006). Cost-effectiveness of screening and referral to an alcohol health worker in alcohol misusing patients attending an accident and emergency department: A decision-making approach. Drug and Alcohol Dependence, 81, 47-54. doi:10.1016/j.drugalcdep.2005.05.015.

Blankers, M., Nabitz, U., Smit, F., Koeter, M. W. J. y Schippers, G. M. (2012). Economic evaluation of internet-based interventions for harmful alcohol use alongside a pragmatic randomized controlled trial. Journal of Medical Internet Research, 14, e134. doi:10.2196/jmir.2052.

Brodtkorb, T-H., Bell, M., Irving, A.H. y Laramée, P. (2016). The cost effectiveness of nalmefene for reduction of alcohol consumption in alcohol-dependent patients with high or very high drinking-risk levels from a UK societal perspective. CNS Drugs, 30, 163-177. doi:10.1007/ s40263-016-0310-2.

Byrnes, J. M., Cobiac, L. J., Doran, C. M., Vos, T. y Shakeshaft, A. P. (2010). Cost-effectiveness of volumetric alcohol taxation in Australia. Medical Journal of Australia, 192, 439-443. doi:10.5694/j.1326-5377.2010.tb03581.x.
Chisholm, D., Moro, D., Bertram, M., Pretorius, C., Gmel, G., Shield, K. y Rehm, J. (2018). Are the "best buys" for alcohol control still valid? An update on the comparative cost-effectiveness of alcohol control strategies at the global level. Journal of Studies on Alcohol and Drugs, 79, 514-522.

Chisholm, D., Rehm, J., Van Ommeren, M. y Monteiro, M. (2004). Reducing the global burden of hazardous alcohol use: a comparative cost-effectiveness analysis. Journal of Studies on Alcohol, 65, 782-793. doi:10.15288/ jsa.2004.65.782.

Cobiac, L., Vos, T., Doran, C. y Wallace, A. (2009). Cost-effectiveness of interventions to prevent alcohol-related disease and injury in Australia. Addiction, 104, 1646-1655. doi:10.1111/j.1360-0443.2009.02708.x.

Cordovilla-Guardia, S., Ortega-Ortega, M., Epstein, D., Fernández-Mondéjar, E. y Vilar-López, R. (2020). Economic analysis of brief motivational intervention following trauma related to drugs and alcohol. Nursing Research, 69, 358-366. doi:10.1097/NNR.0000000000000455.

Corry, J., Sanderson, K., Issakidis, C., Andrews, G. y Lapsley, H. (2004). Evidence-based care for alcohol use disorders is affordable. Journal of Studies on Alcohol, 65, 521-529. doi:10.15288/jsa.2004.65.521.

Coulton, S., Bland, M., Crosby, H., Dale, V., Drummond, C., Godfrey, C.,...Wu, Q. (2017). Effectiveness and cost-effectiveness of opportunistic screening and stepped-care interventions for older alcohol users in primary care. Alcohol and Alcoholism, 52, 655-664. doi:10.1093/alcalc/agx065.

Cowell, A. J., Brown, J. M., Mills, M. J., Bender, R. H. y Wedehase, B. J. (2012). Cost-effectiveness analysis of motivational interviewing with feedback to reduce drinking among a sample of college students. Journal of Studies on Alcohol and Drugs, 73, 226-37. doi:10.15288/ jsad.2012.73.226.

Crawford, M. J., Sanatinia, R., Barrett, B., Byford, S., Dean, M., Green, J.,... Lingford-Hughes, A. (2015). The clinical and cost-effectiveness of brief advice for excessive alcohol consumption among people attending sexual health clinics: A randomised controlled trial. Sexually Transmitted Infections, 91, 37-43. doi:10.1136/sextrans-2014-051561.

Deluca, P., Coulton, S., Alam, M. F., Boniface, S., Donoghue, K., Gilvarry, E.,...Drummond, C. (2020). Screening and brief interventions for adolescent alcohol use disorders presenting through emergency departments: a research programme including two RCTs. Southampton (UK): National Iinstitute for Health Research (NIHR) Journals Library.. doi:10.3310/pgfar08020

Drost, R. M., Paulus, A. T., Jander, A. F., Mercken, L., de Vries, H., Ruwaard, D. y Evers, S. M. (2016). A web-based computer-tailored alcohol prevention program for adolescents: Cost-effectiveness and intersectoral costs 
and benefits. Journal of Medical Internet Research, 18, e93. doi:10.2196/jmir.5223.

Drummond, C., Coulton, S., James, D., Godfrey, C., Parrott, S., Baxter, J.,... Peters, T. (2009). Effectiveness and cost-effectiveness of a stepped care intervention for alcohol use disorders in primary care: pilot study. British Journal of Psychiatry, 195, 448-456. doi:10.1192/bjp. bp.108.056697.

Dunlap, L. J., O’Farrell, T. J., Schumm, J. A., Orme, S. S., Murphy, M. y Murchowski, P. M. (2020). Group versus standard behavioral couples' therapy for alcohol use disorder patients: Cost-effectiveness. Journal of Studies on Alcohol and Drugs, 81, 152-163. doi:10.15288/ jsad.2020.81.152.

Dunlap, L. J., Zarkin, G. A., Bray, J. W., Mills, M., Kivlahan, D. R., McKay, J. R.,...Tonigan J. S. (2010). Revisiting the cost-effectiveness of the COMBINE study for alcohol dependent patients: the patient perspective. Medical Care, 48, 306-313. doi:10.1097/mlr.0b013e3181ca3d40.

Gentilello, L. M., Ebel, B. E., Wickizer, T. M., Salkever, D. S. y Rivara, F. P. (2005). Alcohol interventions for trauma patients treated in emergency departments and hospitals: A cost benefit analysis. Annals of Surgery, 241, 541550. doi:10.1097/01.sla.0000157133.80396.1c.

Giles, E. L., McGeechan, G. J., Coulton, S., Deluca, P., Drummond, C., Howel, D.,... Newbury-Birch, D. (2019). Brief alcohol intervention for risky drinking in young people aged 14-15 years in secondary schools: the SIPS JR-HIGH $R C T$. Southampton (UK): National Iinstitute for Health Research (NIHR) Journals Library.

Havard, A., Shakeshaft, A. P., Conigrave, K. M. y Doran, C. M. (2012). Randomized controlled trial of mailed personalized feedback for problem drinkers in the emergency department: The short-term impact. Alcoholism: Clinical and Experimental Research, 36, 523-531. doi:10.1111/j.1530-0277.2011.01632.x.

Holm, A. L., Veerman, L., Cobiac, L., Ekholm, O. y Diderichsen, F. (2014a). Cost-effectiveness of changes in alcohol taxation in Denmark: A modelling study. Cost Effectiveness and Resource Allocation, 12, 1-13. doi:10.1186/1478-7547-12-1.

Holm, A. L., Veerman, L., Cobiac, L., Ekholm, O. y Diderichsen, F. (2014b). Cost-effectiveness of preventive interventions to reduce alcohol consumption in Denmark. PLoS One, 9, e88041. doi:10.1371/journal. pone.0088041.

Hunter, R., Wallace, P., Struzzo, P., Vedova, R. D., Scafuri, F., Tersar, C.,... Freemantle, N. (2017). Randomised controlled non-inferiority trial of primary care-based facilitated access to an alcohol reduction website: Cost-effectiveness analysis. BMJ Open, 7, e014577. doi:10.1136/ bmjopen-2016-014577.

Ingels, J. B., Corso, P. S., Kogan, S. M. y Brody, G. H. (2013). Cost-effectiveness of the strong African Ameri- can families-teen program: 1-year follow-up. Drug and Alcohol Dependence, 133, 556-561. doi:10.1016/j.drugalcdep.2013.07.036.

Kruger, J., Brennan, A., Strong, M., Thomas, C., Norman, P. y Epton, T. (2014). The cost-effectiveness of a theory-based online health behaviour intervention for new university students: An economic evaluation. $B M C$ Public Health, 14, 1011. doi:10.1186/1471-2458-14-1011.

Kunz, F. M., French, M. T. y Bazargan-Hejazi, S. (2004). Cost-effectiveness analysis of a brief intervention delivered to problem drinkers presenting at an inner-city hospital emergency department. Journal of Studies on Alcohol, 65, 363-370. doi:10.15288/jsa.2004.65.363.

Lai, T., Habicht, J., Reinap, M., Chisholm, D. y Baltussen, R. (2007). Costs, health effects and cost-effectiveness of alcohol and tobacco control strategies in Estonia. Health Policy, 84, 75-88. doi:10.1016/j.healthpol.2007.02.012.

Laramée, P., Bell, M., Irving, A. y Brodtkorb, T. H. (2016). The cost-effectiveness of the integration of nalmefene within the UK healthcare system treatment pathway for alcohol dependence. Alcohol and Alcoholism, 51, 283-290. doi:10.1093/alcalc/agv140.

Laramée, P., Brodtkorb, T.-H., Rahhali, N., Knight, C., Barbosa, C., Francois,... Rehm, J. (2014). The cost-effectiveness and public health benefit of nalmefene added to psychosocial support for the reduction of alcohol consumption in alcohol-dependent patients with high/very high drinking risk levels: A Markov model. BMJ Open, 4, e005376. doi:10.1136/bmjopen-2014-005376.

Li, T., Waters, T. M., Kaplan, E. K., Kaplan, C. M., Nyarko, K. A., Derefinko, K. J.,... Klesges, R. C. (2017). Economic analyses of an alcohol misconduct prevention program in a military setting. Military Medicine, 182, e1562e1567. doi:10.7205/MILMED-D-16-00098.

Millier, A., Laramée, P., Rahhali, N., Aballéa, S., Daeppen, J. B., Rehm, J. y Toumi, M. (2017). Cost-effectiveness of nalmefene added to psychosocial support for the reduction of alcohol consumption in alcohol-dependent patients with high/very high drinking risk levels: A microsimulation model. Journal of Studies on Alcohol and Drugs, 78, 867-876. doi:10.15288/jsad.2017.78.867.

Moore, S.C., Allen, D., Amos, Y., Blake, J., Brennan, A., Buykx, P.,...Young, T. (2020). Evaluating alcohol intoxication management services: the EDARA mixed-methods study. Southampton (UK): National Iinstitute for Health Research (NIHR) Journals Library.

Moraes, E., Campos, G. M., Figlie, N. B., Laranjeira, R. y Ferraz, M. B. (2010). Cost-effectiveness of home visits in the outpatient treatment of patients with alcohol dependence. European Addiction Research, 16, 69-77. doi:10.1159/000268107.

Mortimer, D. y Segal, L. (2005). Economic evaluation of interventions for problem drinking and alcohol depen- 
dence: Cost per QALY estimates. Alcohol and Alcoholism, 40, 549-555. doi:10.1093/alcalc/agh192.

Nadkarni, A., Weiss, H. A., Velleman, R., McCambridge, J., McDaid, D., Park, A. L.,... Patel, V. (2019). Feasibility, acceptability and cost-effectiveness of a brief, lay counsellor-delivered psychological treatment for men with alcohol dependence in primary care: An exploratory randomized controlled trial. Addiction, 114, 1192-1203. doi:10.1111/add.14630.

Nadkarni, A., Weiss, H. A., Weobong, B., McDaid, D., Singla, D. R., Park, A. L.,... Patel, V. (2017a). Sustained effectiveness and cost-effectiveness of Counselling for Alcohol Problems, a brief psychological treatment for harmful drinking in men, delivered by lay counsellors in primary care: 12-month follow-up of a randomised controlled trial. PLoS Medicine, 14, e1002386. doi:10.1371/ journal.pmed.1002386.

Nadkarni, A., Weobong, B., Weiss, H. A., McCambridge, J., Bhat, B., Katti, B.,... Patel, V. (2017b). Counselling for Alcohol Problems (CAP), a lay counsellor-delivered brief psychological treatment for harmful drinking in men, in primary care in India: A randomised controlled trial. Lancet, 389, 186-195. doi:10.1016/S01406736(16)31590-2.

Navarro, H. J., Shakeshaft, A., Doran, C. M. y Petrie, D. J. (2011). The potential cost-effectiveness of general practitioner delivered brief intervention for alcohol misuse: Evidence from rural Australia. Addictive Behaviors, 36, 1191-1198. doi:10.1016/j.addbeh.2011.07.023.

Neighbors, C. J., Barnett, N. P., Rohsenow, D. J., Colby, S. M. y Monti, P. M. (2010). Cost-effectiveness of a motivational intervention for alcohol-involved youth in a hospital emergency department. Journal of Studies on Alcohol and Drugs, 71, 384-394. doi:10.15288/jsad.2010.71.384.

Olmstead, T. A., Graff, F. S., Ames-Sikora, A., McCrady, B. S., Gaba, A. y Epstein, E. E. (2019). Cost-effectiveness of individual versus group female-specific cognitive behavioral therapy for alcohol use disorder. Journal of Substance Abuse Treatment, 100, 1-7. doi:10.1016/j. jsat.2019.02.001.

Palmer, A., Neeser, K., Weiss, C., Brandt, A., Comte, S. y Fox, M. (2000). The long-term cost-effectiveness of improving alcohol abstinence with adjuvant acamprosate. Alcohol and Alcoholism, 35, 478-492. doi:10.1093/ alcalc/35.5.478.

Purshouse, R. C., Brennan, A., Rafia, R., Latimer, N. R., Archer, R. J., Angus, C. R., ... Meier, P. S. (2013). Modelling the cost-effectiveness of alcohol screening and brief interventions in primary care in England. Alcohol and Alcoholism, 48, 180-188. doi:10.1093/alcalc/ags103.

Reddy, V. K., Girish, K., Lakshmi, P., Vijendra, R., Kumar, A. y Harsha, R. (2014). Cost-effectiveness analysis of baclofen and chlordiazepoxide in uncomplicated alcohol-wi- thdrawal syndrome. Indian Journal of Pharmacology, 46, 372-377. doi:10.4103/0253-7613.135947.

Robinson, E., Nguyen, P., Jiang, H., Livingston, M., Ananthapavan, J., Lal, A. y Sacks, G. (2020). Increasing the price of alcohol as an obesity prevention measure: The potential cost-effectiveness of introducing a uniform volumetric tax and a minimum floor price on alcohol in Australia. Nutrients, 12, 603. doi:10.3390/nu12030603.

Rychlik, R., Siedentop, H., Pfeil, T. y Daniel, D. (2003). Cost-effectiveness of adjuvant treatment with acamprosate in maintaining abstinence in alcohol dependent patients. European Addiction Research, 9, 59-64. doi:10.1159/000068810.

Schädlich, P. K. y Brecht, J. G. (1998). The cost effectiveness of acamprosate in the treatment of alcoholism in Germany: Economic evaluation of the prevention of relapse with acamprosate in the management of alcoholism (PRAMA) study. PharmacoEconomics, 13, 719-730. doi:10.2165/00019053-199813060-00008.

Schulz, D. N., Smit, E. S., Stanczyk, N. E., Kremers, S. P., de Vries, H. y Evers, S. M. (2014). Economic evaluation of a web-based tailored lifestyle intervention for adults: Findings regarding cost-effectiveness and cost-utility from a randomized controlled trial. Journal of Medical Internet research, 16, e91. doi:10.2196/jmir.3159.

Slattery, J., Chick, J., Cochrane, M., Craig, J., Godfrey, C., Macpherson, K. y Parrot, S. (2002). Health Technology Assessment of Prevention of Relapse in Alcohol Dependence. Consultation Assessment Report. Scotland: Health Technology Board for Scotland. Recuperado de https://www.drugsandalcohol.ie/19424/1/Health_Technology_Assessment_Prevention_of_relapse_in_alcohol_dependence. pdf.

Sluiter, R. L., Kievit, W., van der Wilt, G. J., Schene, A. H., Teichert, M., Coenen, M. y Schellekens, A. (2018). Cost-effectiveness analysis of genotype-guided treatment allocation in patients with alcohol use disorders using naltrexone or acamprosate, using a modeling approach. European Addiction Research, 24, 245-254. doi:10.1159/000494127.

Smit, F., Lokkerbol, J., Riper, H., Majo, M. C., Boon, B. y Blankers, M. (2011). Modeling the cost-effectiveness of health care systems for alcohol use disorders: How implementation of ehealth interventions improves cost-effectiveness. Journal of Medical Internet Research, 13, e56. doi:10.2196/jmir.1694.

Solberg, L. I., Maciosek, M. V. y Edwards, N. M. (2008). Primary care intervention to reduce alcohol misuse. Ranking its health impact and cost effectiveness. American Journal of Preventive Medicine, 34, 143-152. doi:10.1016/j. amepre.2007.09.035.

Sumnall, H., Agus, A., Cole, J., Doherty, P., Foxcroft, D., Harvey, S.,... Percy, A. (2017). Steps Towards Alcohol Misuse Prevention Programme (STAMPP): A school- and commu- 
nity-based cluster randomised controlled trial. Southhampton (UK): National Iinstitute for Health Research (NIHR) Journals Library.. doi:10.3310/phr05020.

Tariq, L., van den Berg, M., Hoogenveen, R. T. y van Baal, P. H. M. (2009). Cost-effectiveness of an opportunistic screening programme and brief intervention for excessive alcohol use in primary care. PLoS One, 4, e5696. doi:10.1371/journal.pone.0005696.

Torfs, K. y De Graeve, D. (1991). A cost-effectiveness analysis of AOTAL, a drug used to prevent relapse in weaned alcoholics. SESO Working Papers, 1991003. Belgium: University of Antwerp, Faculty of Business and Economics. UKATT Research Team. (2005). Cost effectiveness of treatment for alcohol problems: Findings of the randomised UK alcohol treatment. BMJ, 331, 544-548. doi:10.1136/ bmj.331.7516.544.

Van den Berg, M., van Baal, P. H., Tariq, L., Schuit, A. J., de Wit, G. A. y Hoogenveen, R. T. (2008). The cost-effectiveness of increasing alcohol taxes: a modelling study. BMC Medicine, 6, 36. doi:10.1186/1741-7015-6-36.

Watson, J. M., Crosby, H., Dale, V. M., Tober, G., Wu, Q., Lang, J.,... AESOPS Trial Team (2013). AESOPS: A randomised controlled trial of the clinical effectiveness and cost-effectiveness of opportunistic screening and stepped care interventions for older hazardous alcohol users in primary care. Health Technology Assessment, 17, 1-158. doi:10.3310/hta17250.

Weisner, C., Mertens, J., Parthasarathy, S., Moore, C., Hunkeler, E. M., Hu, T. y Selby, J. V. (2000). The outcome and cost of alcohol and drug treatment in an HMO: Day hospital versus traditional outpatient regimens. Health Services Research, 35, 791-812.

Wutzke, S. E., Shiell, A., Gomel, M. K. y Conigrave, K. M. (2001). Cost effectiveness of brief interventions for reducing alcohol consumption. Social Science $\mathcal{E}$ Medicine, 52, 863-870. doi:10.1016/s0277-9536(00)00189-1.

Zarkin, G. A., Bray, J. W., Aldridge, A., Mitra, D., Mills, M. J., Couper, D. J. y Cisler, R. A. (2008). Cost and cost-effectiveness of the COMBINE study in alcohol-dependent patients. Archives of General Psychiatry, 65, 1214-1221. doi:10.1001/archpsyc.65.10.1214. 